Draft Version OCtober 29, 2018

Preprint typeset using $\mathrm{LAT}_{\mathrm{E}} \mathrm{X}$ style emulateapj v. 08/22/09

\title{
A LARGE RESERVOIR OF IONIZED GAS IN THE GALACTIC HALO: IONIZED SILICON IN HIGH-VELOCITY AND INTERMEDIATE-VELOCITY CLOUDS
}

\author{
J. Michael Shull, Jennifer R. Jones ${ }^{1}$, Charles W. Danforth, \& Joseph A. Collins ${ }^{2}$ \\ CASA, Department of Astrophysical and Planetary Sciences, \\ University of Colorado, 389-UCB, Boulder, CO 80309 \\ michael.shull@colorado.edu,charles.danforth@colorado.edu, jcollins@casa.colorado.edu \\ Draft version October 29, 2018
}

\begin{abstract}
The low Galactic halo is enveloped by a sheath of ionized, low-metallicity gas, which can provide a substantial $\left(1 M_{\odot} \mathrm{yr}^{-1}\right)$ cooling inflow to replenish star formation in the disk. Using absorption spectra from the Hubble Space Telescope and Far Ultraviolet Spectroscopic Explorer toward 37 active galactic nuclei at high latitude, we detect widespread interstellar Si III $\lambda 1206.50$ absorption: 61 highvelocity clouds (HVCs) along 30 sight lines and 22 intermediate-velocity clouds (IVCs) along 20 sight lines. We find a segregation of redshifted and blueshifted absorbers across the Galactic rotation axis at $\ell=180^{\circ}$, consistent with a lag in the rotation velocity above the Galactic plane. The HVC sky coverage is large ( $81 \pm 5 \%$ for 30 out of 37 directions) with Si III optical depth typically 4-5 times that of O VI $\lambda 1031.926$. The mean HVC column density per sight line, $\left\langle\log \mathrm{N}_{\mathrm{SiIII}}\right\rangle=13.42 \pm 0.21$, corresponds to total column density $\mathrm{N}_{\mathrm{HII}} \approx\left(6 \times 10^{18} \mathrm{~cm}^{-2}\right)\left(Z_{\mathrm{Si}} / 0.2 Z_{\odot}\right)^{-1}$ of ionized low-metallicity gas, similar to that inferred in O VI. This reservoir could total $10^{8} M_{\odot}$ and produce a mass infall rate $\sim 1 M_{\odot} \mathrm{yr}^{-1}$. If Si II, Si III, Si IV, and H I are co-spatial and in photoionization equilibrium, the mean photoionization parameter in the low halo, $\langle\log U\rangle=-3.0_{-0.4}^{+0.3}$, approximately ten times lower than observed in the low-redshift intergalactic medium. The metallicities in some HVCs, derived from [Si II/H I], are 10-30\% solar, whereas values found from all three silicon ions are lower in the pure-photoionization models. These formally lower metallicities are highly uncertain, since some of the higher ions may be collisionally ionized. The HVC and IVC metallicities may be compared with the median photometric metallicity, $[\mathrm{Fe} / \mathrm{H}]=-1.46 \pm 0.30$, for $\sim 200,000$ halo $\mathrm{F} / \mathrm{G}$ stars in the Sloan Digital Sky Survey.

Subject headings: ISM: clouds — Galaxy: halo — Galaxy: abundances — ultraviolet: general
\end{abstract}

\section{INTRODUCTION}

Interstellar gas above the plane of the Milky Way disk has been studied through a number of techniques, including emission in $\mathrm{H} \mathrm{I}(21 \mathrm{~cm})$, soft X-rays, and diffuse $\mathrm{H} \alpha$, pulsar dispersion measures, and absorption lines in the optical, ultraviolet, and X-ray. These observations suggest a multiphase gaseous medium in the low halo, consisting of high-latitude absorption-line clouds, pressureconfined by a hot (coronal) gaseous background. One of the first hints of this hot halo came from the detection of high-latitude interstellar clouds in 21-cm emission (Oort 1966) and in Na I and Ca II absorption (Adams 1949; Münch \& Zirin 1961; Hobbs 1965). In order to provide for cloud confinement, Spitzer (1956) proposed the existence of a hot, ionized "Galactic corona" of $8 \mathrm{kpc}$ vertical extent, with temperature $T \approx 10^{6} \mathrm{~K}$ and hydrogen density $n_{H} \approx 5 \times 10^{-4} \mathrm{~cm}^{-3}$.

With the advent of ultraviolet spectrographs aboard the International Ultraviolet Explorer (IUE), the Hubble Space Telescope (HST), and the Far Ultraviolet Spectroscopic Explorer (FUSE), astronomers gained access to sensitive diagnostics of hot halo gas, particularly the far-UV resonance lines of high-ionization species such as C IV, Si IV, N V, and O VI (Savage \& Sembach 1991;

\footnotetext{
1 Now at Department of Physics \& Astronomy, Michigan State University, East Lansing, MI 48824, westbr39@msu.edu

2 Also at Front Range Community College, Larimer Campus, 4616 S. Shields St., Fort Collins, CO 80526
}

Shull \& Slavin 1994; Savage et al. 2003; Indebetouw \& Shull 2004; Collins, Shull, \& Giroux 2003, 2007; Fox, Savage, \& Wakker 2006). Recent low-resolution spectra of active galactic nuclei (AGN) taken with Chandra and $X M M$-Newton telescopes have detected X-ray absorption lines at redshifts $z \approx 0$ (Nicastro et al. 2002; Fang et al. 2002; McKernan et al. 2004) that may arise from hot gas in the Galactic halo or Local Group. These X-ray absorbers include lines from O VII (21.602 $\AA)$, O VIII $(18.969 \AA), \mathrm{N}$ VI $(28.787 \AA), \mathrm{N}$ VII $(24.782 \AA)$, and C VI $(33.776 \AA)$. Owing to the low resolution of the X-ray spectrographs, the location of these " $z=0$ absorbers" remains controversial, possibly residing in a Galactic halo of radial extent $r \sim 20 \mathrm{kpc}$ (Bregman \& Lloyd-Davies 2007) or in a thick gaseous disk, of vertical scale height $h \sim 2 \mathrm{kpc}$ (Yao \& Wang 2007; Yao et al. 2008).

In UV/optical spectra, the observed ion stages in highlatitude clouds range from low-ionization states (H I, O I, $\mathrm{S}$ II, Si II, Na I, Ca II) to ionized gas traced by C III, C IV, N V, O VI, Si III, and Si IV. These "high ions" could arise from both photoionization and collisional ionization, depending on cloud proximity to sources of ionizing photons or interfaces with hot gas. They are best traced by absorption in the ultraviolet lines of $\mathrm{O}$ VI $(\lambda 1031.926,1037.617), \mathrm{N} \mathrm{V}(\lambda 1238.821,1242.802)$, and C IV $(\lambda 1548.195,1550.770)$. Ultraviolet surveys of the vertical distribution of these high ions suggest a layer of hot gas, with scale heights ranging from $3-5 \mathrm{kpc}$ 
(Sembach \& Savage 1992; Shull \& Slavin 1994). This corona is expected to lie near the virial temperature, $T_{\mathrm{vir}} \approx\left(2 \times 10^{6} \mathrm{~K}\right) M_{12}^{2 / 3}\left[\left(1+z_{\mathrm{vir}}\right) / 4\right]$ for the Milky Way, with total mass $M=\left(10^{12} M_{\odot}\right) M_{12}$ assembled at virialization redshift $z_{\text {vir }} \approx 3$. Laced with heavy elements, this gas is expected to cool into clumps (Maller \& Bullock 2004; Collins, Shull, \& Giroux 2004), fall inward, and intercept uprising gas from superbubbles in the Galactic disk. The result is a disk-halo interface and Galactic fountain (Shapiro \& Field 1976; Bregman 1980; Houck \& Bregman 1990; Shull \& Slavin 1994). It has been suggested that $\mathrm{O}$ VI and $\mathrm{N} \mathrm{V}$ may reside in bow shocks and other interfaces between infalling clouds and the hot gaseous corona (Indebetouw \& Shull 2004; Fox et al. 2006)

In many disk galaxies, the infall of low-metallicity gas seems to be important for gas replenishment, the evolution of star formation rates, and the chemical imprint on the mass-metallicity relations (Tremonti et al. 2004; Erb et al. 2006). In the Milky Way, the processes of gaseous infall and recycling are observed in Galactic high-velocity clouds (HVCs) and intermediate velocity clouds (IVCs), defined as clouds of primarily neutral hydrogen whose velocities are inconsistent with the standard model of Galactic rotation. The distinction between HVCs and IVCs lies in their local-standard-of-rest (LSR) velocities, with a nominal population break at $\left|V_{\mathrm{LSR}}\right|=90-100$ $\mathrm{km} \mathrm{s}^{-1}$. Following the convention of Wakker \& van Woerden (2007), we define IVCs to lie between $\left|v_{\mathrm{LSR}}\right| \approx$ $30-90 \mathrm{~km} \mathrm{~s}^{-1}$ and HVCs to have $\left|v_{\mathrm{LSR}}\right|>90 \mathrm{~km} \mathrm{~s}^{-1}$. By analyzing the metallicity of this low-halo gas, we can constrain their spatial location and origin. Galactic fountains are expected to be metal-rich, so the resulting absorbers should have metallicities near solar. By contrast, gas falling into the disk from the Local Group or intergalactic medium (IGM) would be metal-poor, perhaps $10-30 \%$ of solar values.

Two interpretations have arisen for the HVC and IVC positions in the Galaxy. The first model places HVCs in the low Galactic halo (Collins et al. 2003; Tripp et al. 2003; Fox et al. 2006) as inflows onto and outflows from the disk. The second model identifies a sub-class of compact HVCs, at locations in the Local Group (Blitz et al. 1999). Constraining the ionization conditions may distinguish whether HVCs are similar to gas from the Galactic disk, within the Local Group, or in the IGM. This uncertainty in HVC distances to HVCs creates a semantic problem in defining their location in the "thick disk" (scale height $h \leq 1 \mathrm{kpc}$ ) or "low halo" ( $h \approx 5$ $10 \mathrm{kpc}$ ). Distance measurements have recently been reported for several HVCs, using the presence or absence of Ca II absorption toward Galactic halo stars. These studies bracket the distance to HVC Complex C at $10 \pm 2.5$ kpc (Thom et al. 2008; Wakker et al. 2007) and place other HVCs distances at $\sim 10 \mathrm{kpc}$ (Wakker et al. 2008). Complex $\mathrm{C}$ is a good example of infalling metal-poor gas (Wakker et al. 1999; Collins et al. 2003, 2007; Tripp et al. 2003). By analyzing [O I/H I] along 11 AGN sight lines through Complex C, Collins et al. (2007) find a column-density weighted metallicity of $0.13 Z_{\odot}$. Toward PG 1259+593, another AGN sight line through Complex $\mathrm{C}$, the IVC has a near-solar $[\mathrm{O} \mathrm{I} / \mathrm{H} \mathrm{I}]$ abundance, while the HVC has an abundance $\sim 10 \%$ solar (Richter et al. 2001; Sembach et al. 2004; Collins et al. 2007). These observations suggest that IVCs are material near the Galactic plane, while HVCs have fallen in from more distant gas.

Many groups have used data from HST, FUSE, and the Leiden/Dwingeloo Survey (LDS) to derive column densities of a variety of interstellar HVC species. Savage et al. (2003) studied the distribution and kinematics of $\mathrm{O}$ VI in the Galactic halo, using FUSE spectra of O VI over the velocity range $-100<v_{\mathrm{LSR}}<+100 \mathrm{~km} \mathrm{~s}^{-1}$, along paths toward 100 AGN and two distant halo stars. Sembach et al. (2003) studied O VI HVCs in the same 102 sight lines, identifying 84 high-velocity features at $-500<v_{\mathrm{LSR}}<+500 \mathrm{~km} \mathrm{~s}^{-1}$, with mean column density $\left\langle\log N_{\text {OVI }}\right\rangle=13.95 \pm 0.34$. These O VI absorbers exhibit velocity centroids ranging from -372 to +385 $\mathrm{km} \mathrm{s}^{-1}$ and line widths, expressed as doppler velocities, of $b_{\text {OVI }}=16-72 \mathrm{~km} \mathrm{~s}^{-1}$. High-velocity O VI was seen along 59 of 102 sight lines, suggesting that $\sim 60 \%$ of the sky, and perhaps even higher controlling for data-quality, is covered by high-velocity ionized gas. This covering fraction is higher than that seen in 21-cm emission, measured at $37 \%$ for column densities $N_{\mathrm{HI}} \geq 7 \times 10^{17} \mathrm{~cm}^{-2}$ (Murphy, Lockman, \& Savage 1995).

In this paper, we focus on silicon in three ionization states accessible to UV spectroscopy with $H S T$ and FUSE. We analyze multiple strong ultraviolet absorption features, including Si II (1020.70, 1190.42, 1193.29, $1260.42,1304.37,1526.71 \AA)$, Si III $(1206.50 \AA)$, and Si IV (1393.76, $1402.77 \AA$ ). As background targets (Figure 1) we chose 37 AGN distributed over high Galactic latitudes, $|b| \geq 20^{\circ}$, to avoid confusion from absorption in the Galactic plane. Our previous spectroscopic studies of HVCs (Gibson et al. 2000, 2001; Collins et al. 2003, 2007) compared ions of many elements (C, N, O, $\mathrm{Si}, \mathrm{S}, \mathrm{Fe}$ ) in Complex $\mathrm{C}$ and the Magellanic Stream, although $[\mathrm{O} \mathrm{I} / \mathrm{H} \mathrm{I}]$ is the best indicator of metallicity, owing to charge-exchange coupling. Assumptions about the relative metallicity between elements $(\mathrm{O}, \mathrm{Si}, \mathrm{S}, \mathrm{Fe})$ can be bypassed by observing multiple ionization states of the same element. In the ultraviolet, multiple ions are available for silicon (Si II/III/IV), as well as carbon (C II/III/IV), sulfur (S II/III/IV), and iron (Fe II/III).

This paper is organized as follows. In $\S 2$, we discuss the importance of the strong Si III $\lambda 1206.50$ absorption line as a probe of Galactic HVCs and IVCs. We then describe the acquisition and reduction of the $21-\mathrm{cm}$ data from the LDS, and the ultraviolet absorbers (Si II, Si III, Si IV) with FUSE and HST. Our survey found 61 HVCs and 22 IVCs along 37 AGN sight lines. In $\S 3$, we discuss our data analysis and photoionization modeling. In $\S 4$ we discuss our results, placing these observations in the context of other measures of the Galactic halo and lowredshift IGM.

\section{SILICON IONS IN HVCS AND IVCS}

\subsection{The Importance of Si III}

We begin with the strong Si III $\lambda 1206.500$ absorption line, for which we have recently completed an HVC survey (Collins, Shull, \& Giroux 2009). This line has a large oscillator strength, $f=1.63$ (Morton 2003), making it a good tracer of ionized gas, comparable in sensitivity to the O VI doublet $(1031.926,1037.617 \AA$ ). The Si III 


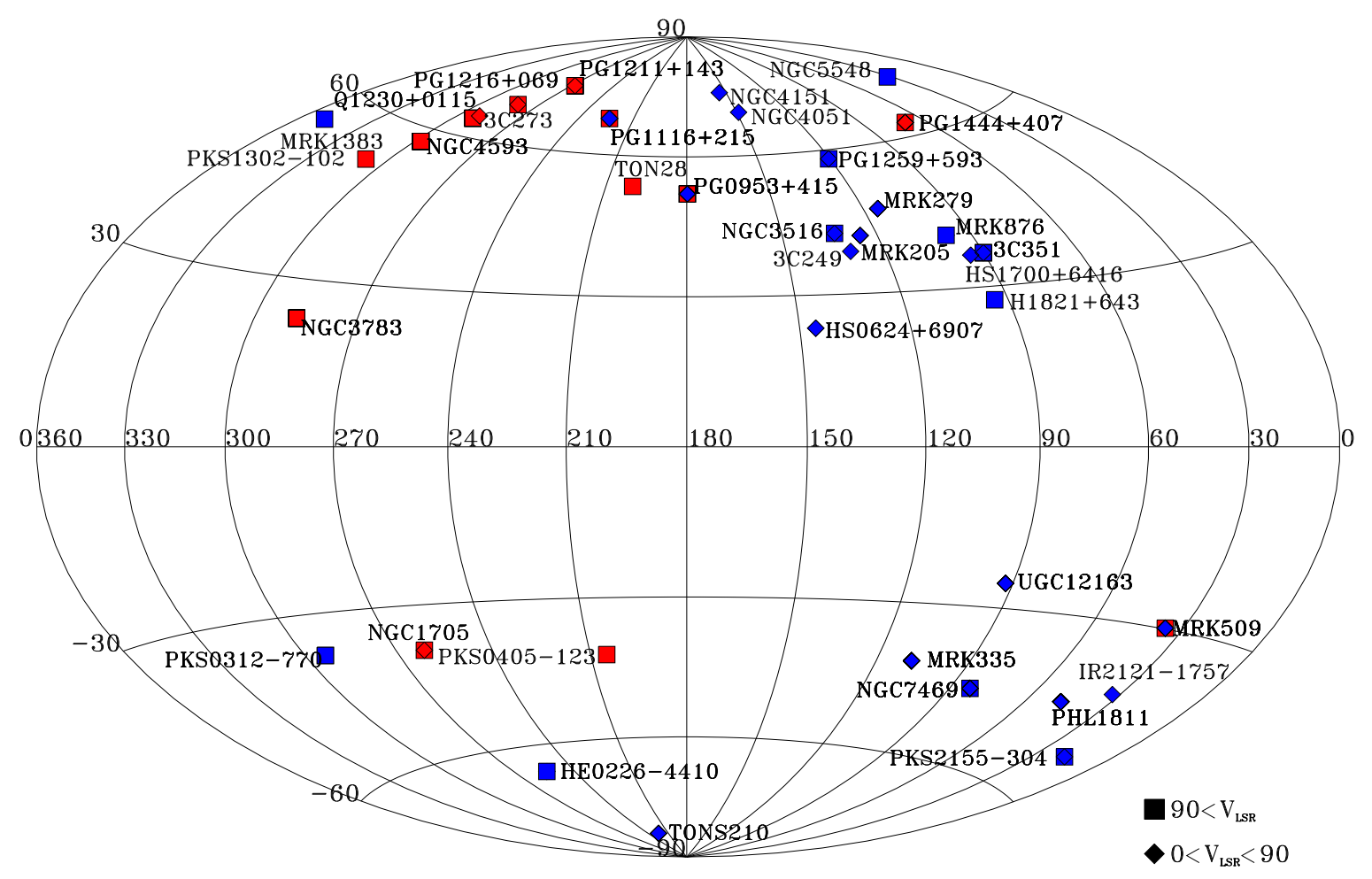

FIG. 1.- Positions of 37 observed AGN sight lines (25 in northern Galactic hemisphere and 12 in south) plotted in Galactic Coordinates, with anticenter $\left(\ell=180^{\circ}\right)$ shown in the middle. Central velocities of HVC and IVC absorption features in each sight line are color-coded as shown in the legend. Of the 37 sight lines, 30 (81\%) showed high-velocity Si III. Multiple HVCs were seen along 20 sight lines $(8$ with three or more HVCs), while 7 sight lines showed no HVCs in Si III (only an IVC). The HVC redshifts and blueshifts are largely segregated on either side of the Galactic rotation axis through $\ell=180^{\circ}$. See text for possible interpretation as a dropoff in circular velocity above the disk.

absorption line is intrinsically 14 times stronger (in $f \lambda$ ) than O VI $\lambda 1031.926$, which compensates for the lower abundance ratio, $(\mathrm{Si} / \mathrm{O})_{\odot} \approx 0.071$ based on solar abundances, $(\mathrm{Si} / \mathrm{H})_{\odot}=3.24 \times 10^{-5}$ and $(\mathrm{O} / \mathrm{H})_{\odot}=4.57 \times$ $10^{-4}$, from Asplund et al. (2005). With STIS/E140M, the Si III line provides sensitivity to column densities at or below $\mathrm{N}_{\mathrm{SiIII}} \approx\left(10^{12} \mathrm{~cm}^{-2}\right) N_{12}$, corresponding to equivalent width $W_{\lambda}=(21.0 \mathrm{~m} \AA) N_{12}$. For a Gaussian profile, the Si III optical depth at line center is $\tau_{0} \approx(0.295) N_{12} b_{10}^{-1}$, scaled to a doppler width $b_{\text {SiIII }}$ $=\left(10 \mathrm{~km} \mathrm{~s}^{-1}\right) b_{10}$, chosen to reflect the turbulence and velocity components that broaden HVC metal-line absorbers (Collins et al. 2003, 2007). Typical HVCs exhibit Si III absorption over an extended velocity range, $\Delta v=40-100 \mathrm{~km} \mathrm{~s}^{-1}$, far broader than any single component would allow. Indeed, the curves of growth derived for low metal ions in HVC Complex $\mathrm{C}$ (Collins et al. 2003, 2007) range from $b \approx 7-18 \mathrm{~km} \mathrm{~s}^{-1}$, consistent with non-thermal line widths. Given these large velocity dispersions, we expect Si III line saturation to set in at column densities $\mathrm{N}_{\mathrm{SiIII}}>\left(3.4 \times 10^{12} \mathrm{~cm}^{-2}\right) b_{10}$ in each STIS/E140M resolution element. In our column-density tables, we express $\log N_{\mathrm{SiIII}}$ as a lower limit when saturation is believed to exist.

To compare the Si III and O VI observations, it is useful to estimate the expected relative absorption-line strengths. The absorption optical depth at frequency $\nu$ scales as $\tau_{\nu} \propto N f \lambda /(\Delta v)$, where $N$ is the column density, $f \lambda$ measures the intrinsic line strength, and $\Delta v$ is the effective line width of the absorption profile. The ratio of optical depths of Si III $\lambda 1206.50$ and O VI $\lambda 1031.93$ is then,

$$
\frac{\tau_{\mathrm{SiIII}}}{\tau_{\mathrm{OVI}}}=(1.02)\left[\frac{Z_{\mathrm{Si}} f_{\mathrm{SiIII}} \mathrm{N}_{\mathrm{H}}^{\mathrm{SiII}} \Delta v_{\mathrm{OVI}}}{Z_{\mathrm{O}} f_{\mathrm{OVI}} \mathrm{N}_{\mathrm{H}}^{\mathrm{OVI}} \Delta v_{\mathrm{SiIII}}}\right]
$$

where $Z_{\mathrm{Si}}$ and $Z_{\mathrm{O}}$ are silicon and oxygen metallicities, $f_{\text {SiIII }}$ and $f_{\text {OVI }}$ are the ionization fractions of these species, $\Delta v_{\mathrm{SiIII}}$ and $\Delta v_{\mathrm{OVI}}$ are the effective line widths of the two lines, and $\mathrm{N}_{\mathrm{H}}^{\mathrm{SiIII}}$ and $\mathrm{N}_{\mathrm{H}}^{\mathrm{OVI}}$ are the hydrogen column densities of the multiphase gas traced by Si III and $\mathrm{O}$ VI respectively. Because the line widths are probably non-thermal, we expect that $\Delta v_{\mathrm{OVI}} / \Delta v_{\text {SiII }} \approx 1$, rather than the higher values suggested by their massdependent thermal doppler widths, $\Delta v_{\mathrm{OVI}} / \Delta v_{\mathrm{SiIII}}=$ $1.32\left(T_{\mathrm{OVI}} / T_{\mathrm{SiIII}}\right)^{1 / 2}$. The ionization fractions, $f_{\mathrm{SiIII}} \approx$ $0.7 \pm 0.2$ and $f_{\text {OVI }} \approx 0.2 \pm 0.1$, are also uncertain and depend on the relative importance of photoionization and collisional ionization. In the multiphase conditions likely in HVCs, these ionization fractions can have wide ranges. However, Si III (a Mg-like ion with a closed-subshell $3 s^{2}$ valence structure) typically has a much larger ionization fraction than O VI (a Li-like ion with a weakly bound $2 s$ valence electron). For example, if Si III and O VI each exist in collisional ionization equilibrium, their peak ionization fractions (Sutherland \& Dopita 1993) would be $f_{\mathrm{SiIII}}=0.90$ at $\log T_{\max }=4.45$ and $f_{\mathrm{OVI}}=0.22$ at $\log T_{\max }=5.45$. Therefore, adopting an ionization ratio $f_{\mathrm{SiIII}} / f_{\mathrm{OVI}} \approx 4$ and setting the other scaling ratios in eq. 
TABLE 1

Total AGN Sight Lines in Survey

\begin{tabular}{|c|c|c|c|c|c|c|}
\hline Sightline & $\begin{array}{l}\mathrm{RA}(\mathrm{J} 2000) \\
(\mathrm{h} \mathrm{m} \mathrm{s})\end{array}$ & 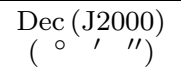 & $\left(\begin{array}{l}\ell \\
\left({ }^{\circ}\right.\end{array}\right)$ & $\left(\begin{array}{l}b \\
\left({ }^{\circ}\right.\end{array}\right)$ & $\begin{array}{l}F U S E \\
(\mathrm{ksec})\end{array}$ & $\begin{array}{r}H S T \\
(\mathrm{ksec})\end{array}$ \\
\hline Mrk 335 & 000619.5 & +201210.3 & 108.76 & -41.42 & 97.0 & 17.1 \\
\hline Ton S210 & 012151.6 & -282057.3 & 224.97 & -83.16 & 52.2 & 27.4 \\
\hline HE $0226-440$ & $02 \quad 2815.2$ & -405716.0 & 253.9 & -65.77 & 64.2 & 3.02 \\
\hline PKS 0312-770 & 031155.4 & -765150.8 & 293.4 & -37.55 & 15.4 & 12.6 \\
\hline PKS 0405-123 & $\begin{array}{lll}04 & 07 & 48.2\end{array}$ & -121131.5 & 204.93 & -41.76 & 71.1 & 27.4 \\
\hline NGC 1705 & 045413.5 & -532139.8 & 261.0 & -38.74 & 15.4 & 3.11 \\
\hline HS $0624+6907$ & 063002.7 & +690503.7 & 145.71 & 23.35 & 112.3 & 62.0 \\
\hline PG $0953+415$ & 095652.4 & +411522.0 & 179.79 & 51.71 & 72.1 & 27.4 \\
\hline Ton 28 & 100402.6 & +285535.5 & 200.08 & 53.21 & 11.2 & 33.0 \\
\hline 3C 249 & 110413.7 & +765858.2 & 130.39 & 38.55 & 216.8 & 68.8 \\
\hline NGC 3516 & 110647.6 & +723406.9 & 133.24 & 42.40 & 75.1 & 20.6 \\
\hline PG $1116+215$ & 111908.7 & +211918.2 & 223.36 & 68.21 & 77.0 & 26.5 \\
\hline NGC 3783 & $\begin{array}{lll}11 & 39 & 01.7\end{array}$ & -374418.9 & 287.4 & 22.94 & 37.3 & 2.7 \\
\hline NGC 4051 & 120309.6 & +443152.8 & 148.8 & 70.08 & 28.8 & 2.2 \\
\hline NGC 4151 & 121032.6 & +392421.0 & 155.08 & 75.06 & 97.8 & 13.2 \\
\hline $\mathrm{PG} 1211+143$ & 121417.6 & +140312.7 & 267.55 & 74.31 & 52.3 & 42.5 \\
\hline PG $1216+069$ & 121920.9 & +063838.4 & 281.0 & 68.14 & 12.4 & 2.90 \\
\hline Mrk 205 & 122144.0 & +751838.3 & 125.45 & 41.67 & 203.6 & 62.1 \\
\hline $3 \mathrm{C} 273$ & 122906.7 & +020308.9 & 289.95 & 64.36 & 42.3 & 18.7 \\
\hline Q $1230+0115$ & 123050.0 & +011521.7 & 291.26 & 63.66 & 4.0 & 9.8 \\
\hline NGC 4593 & 123939.5 & -052038.0 & 297.48 & 57.40 & & 11.0 \\
\hline PG 1259+593 & 130113.1 & +590205.7 & 120.56 & 58.05 & 668.3 & 95.8 \\
\hline PKS $1302-102$ & 130533.0 & -103319.3 & 308.5 & 52.16 & 83.3 & 11.0 \\
\hline Mrk 279 & 135303.4 & +691829.9 & 115.04 & 46.86 & 228.5 & 54.6 \\
\hline NGC 5548 & 141759.5 & +250812.4 & 31.96 & 70.50 & 55.0 & 69.8 \\
\hline Mrk 1383 & 142906.6 & +011706.6 & 349.22 & 55.13 & 63.5 & 10.5 \\
\hline PG $1444+407$ & 144645.9 & +403506.4 & 69.90 & 62.72 & 10.0 & 48.6 \\
\hline Mrk 876 & 161357.2 & +654309.6 & 98.27 & 40.38 & 46.0 & 29.2 \\
\hline HS $1700+6416$ & 170100.6 & +641209.9 & 94.40 & 36.16 & 285.5 & 9.1 \\
\hline 3C 351 & $1704 \quad 41.5$ & +604428.0 & 90.08 & 36.38 & 141.9 & 77.0 \\
\hline H $1821+643$ & 182157.3 & +642036.3 & 94.00 & 27.42 & 132.3 & 50.9 \\
\hline Mrk 509 & $\begin{array}{lll}20 & 44 & 09.7\end{array}$ & -104324.7 & 35.97 & -29.86 & 62.3 & 7.6 \\
\hline IR $2121-1757$ & 212441.7 & -174445.9 & 32.78 & -41.64 & & 5.3 \\
\hline PHL 1811 & 215501.6 & -092226.0 & 47.47 & -44.82 & 75.0 & 33.9 \\
\hline PKS 2155-304 & 215852.1 & $\begin{array}{lll}-30 & 13 & 32.3\end{array}$ & 17.73 & -52.25 & 123.2 & 10.8 \\
\hline UGC 12163 & 224239.3 & +294331.3 & 92.14 & -25.34 & 60.9 & 10.3 \\
\hline NGC 7469 & $\begin{array}{lll}23 & 03 & 15.6\end{array}$ & +085226.2 & 83.10 & -45.47 & 44.3 & 22.8 \\
\hline
\end{tabular}

(1) equal to unity, we expect Si III to be stronger than O VI, with $\tau_{\text {SiIII }} / \tau_{\text {OVI }} \approx 4$.

The above estimate is merely illustrative, since Si III and $\mathrm{O}$ VI probably reside in different spatial and thermal portions of the HVCs. For example, if the HVC is an infalling clump of cooling gas from the halo, the $\mathrm{H}$ I and low ions (Si II, S II, O I) are likely to be a mixture of cool gas at $T<10^{3} \mathrm{~K}$ and photoionized gas at $10^{4} \mathrm{~K}$. The higher ions ( $\mathrm{O}$ VI, N V) may come from bow shocks or interfaces with hotter halo gas and have $T>10^{5} \mathrm{~K}$. The intermediate ions ( $\mathrm{Si}$ III, Si IV, $\mathrm{C}$ III, C IV) may arise in both photoionized and collisionally ionized gas, complicating the ionization corrections needed to derive metallicities. Such environments include bow shocks around infalling HVCs, expanding shells around wind-driven bubbles, galactic winds, or supershells. These multiphase effects, in which Si III and $\mathrm{O}$ VI do not reside in the same gas, are captured in eq. (1) by the ratio, $\mathrm{N}_{\mathrm{H}}^{\mathrm{SiIII}} / \mathrm{N}_{\mathrm{H}}^{\mathrm{OVI}}$.

These considerations suggest that Si III is a strong tracer of ionized gas, probing different thermal phases and ionization states than O VI. Our observations show that Si III $\lambda 1206$ is typically $4-5$ times stronger than O VI $\lambda 1032$, as illustrated in Figure 2 for eight sight lines with HVCs and IVCs. Therefore, Si III provides an excellent probe of low column density (ionized) HVCs, which can be used with O VI, N V, C IV, Si IV, and other ions to trace the content and metallicity of ionized gas at high latitude. One limitation to the single Si III line is the the possibility of line saturation. Without any second Si III transition, it is difficult to use strong $\lambda 1206$ absorption systems, except to define the velocity range and provide ionization information in tandem with $\mathrm{Si}$ II and Si IV. In our survey, we use Si III to identify weak HVCs and rely on Si II and Si IV to separate HVCs into velocity components.

In the next section, we focus on what can be learned assuming that the three silicon ions in HVCs and IVCs are predominantly photoionized. A significant advance in HVC photoionization modeling is enabled by access to three Si ionization stages, allowing us to constrain the photoionization parameter, $U=n_{\gamma} / n_{H}$, where $n_{\gamma}$ is the density of ionizing photons and $n_{H}$ is the density of hydrogen nuclei. Combined with H I column densities, $N_{\mathrm{HI}}$, these parameters enable us to derive physical models of the cloud densities $\left(n_{H}\right)$, elemental abundances, and silicon metallicities, $Z_{\mathrm{Si}}$. ¿From the distribution of ionization parameters and metallicities, we hope to define the spatial location of HVCs and IVCs from the Galactic plane and infer the nucleosynthetic sources of their heavy elements. 


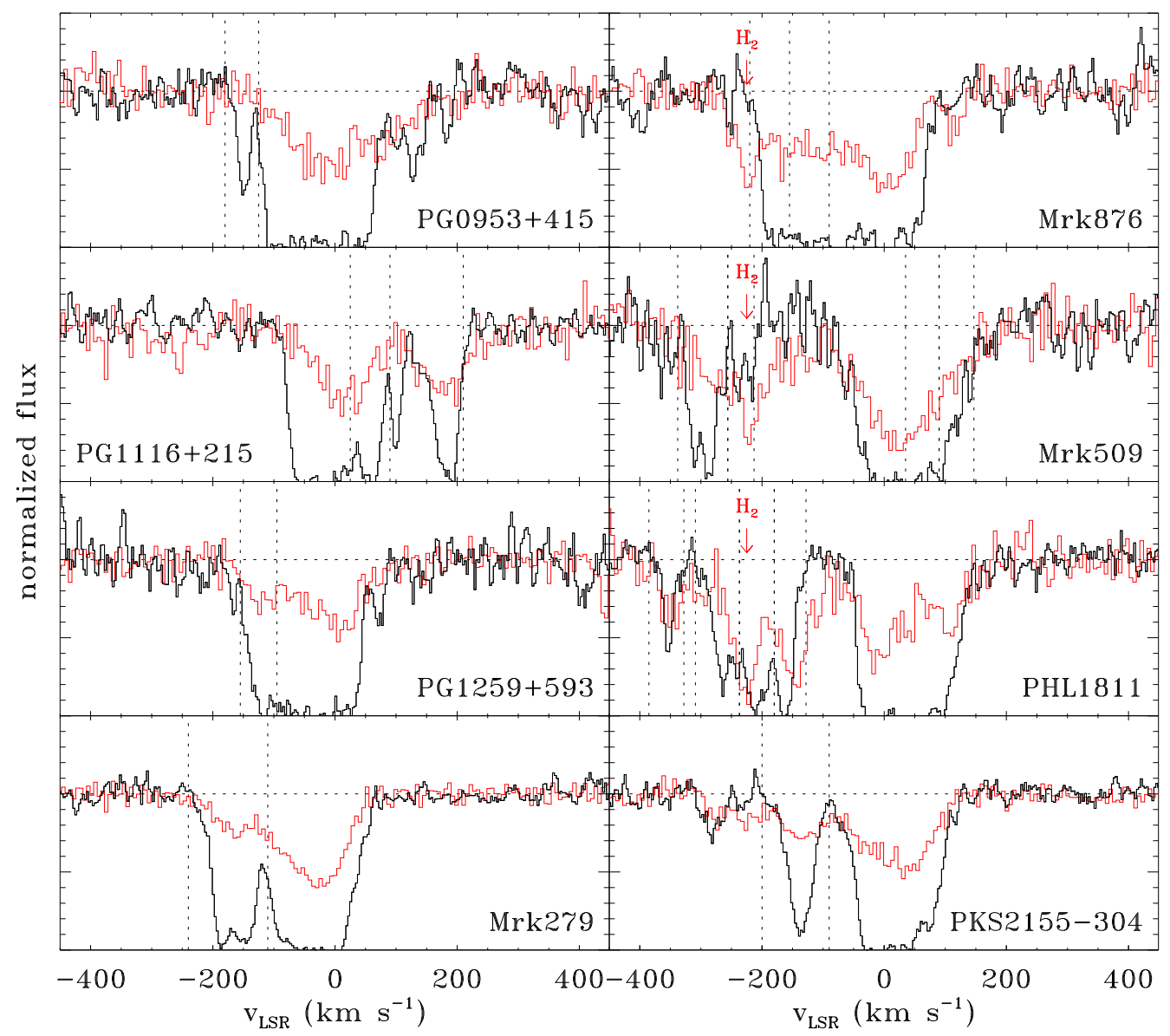

FIG. 2.- Ultraviolet spectra (HST/STIS and FUSE) of 8 AGN, showing absorption spectra of Si III $\lambda 1206.50$ (black) and O VI $\lambda 1031.93$ (red) vs. LSR velocity. Velocity ranges for HVCs and IVCs are noted with vertical dashed lines, as is the position of $\mathrm{H}_{2}$ absorption along 3 sight lines. Note that Si III is typically 4-5 times stronger than O VI in the HVCs, making it an excellent tracer of small amounts of ionized gas.

\subsection{AGN Sight Lines and their HVC/IVC Features}

¿From our database of HST and FUSE spectra of highlatitude AGN (Danforth \& Shull 2005, 2008), we selected 37 sight lines (see Figure 1) to search for the presence of Si II, Si III, and Si IV. These particular targets (Table 1) were selected from a larger sample of 58 AGN (Collins, Shull, \& Giroux 2009) observed with both the E140M echelle and G140M gratings. These 37 sight lines were studied both for the presence of HVCs and IVCs and for the quality of their data. The minimum signal-to-noise per resolution element is $(\mathrm{S} / \mathrm{N})_{\min }=3$, and the mean is $\langle\mathrm{S} / \mathrm{N}\rangle=13$.

The LDS data were analyzed for the $\mathrm{H}$ I emission spectra and column densities, $N_{\mathrm{HI}}$, as described in Wakker et al. (2003) and Tumlinson et al. (2004), having been reduced by Hartmann \& Burton (1997). At first, it appeared that the $\mathrm{H}$ I data would give the best limits for the velocity range of HVCs and IVCs. However, in most cases, the ranges were better determined by silicon absorption features themselves. The strong Si III $\lambda 1206.5$ line is a sensitive tracer of ionized gas, although often saturated. In those cases, the weaker Si II lines usually trace the velocity ranges of the cooler HVC components.

For spectra with Si II, Si III, and Si IV absorption features, we used the Space Telescope Imaging Spectrograph (STIS) echelle on HST. We analyzed STIS data for Si III and Si IV lines in all 83 absorbers $(61 \mathrm{HVCs}$ and 22 IVCs) and for Si II lines. In some HVC/IVCs, we also analyzed FUSE data for Si II $\lambda 1020.699$. Some of these absorbers are "highly ionized HVCs", originally defined (Sembach et al. 1995, 1999) through the presence of C IV or O VI absorption, but with no H I 21-cm emission. For example, the sight lines to PKS 2155-304 and Mrk 509 exhibit several of this absorber type, in which the $\mathrm{HVC}$ is detected in high ions (C IV, O VI) but not in $\mathrm{H} \mathrm{I}$ emission. Many of these highly ionized HVCs are detected in $\mathrm{H}$ I through their Lyman-series absorption lines and in low ions such as O I, C II, Si II, and S II (Collins et al. 2004, 2005). These absorbers are probably an extension of the HVC population to column densities, $N_{\mathrm{HI}} \leq 10^{18} \mathrm{~cm}^{-2}$, below the sensitivity level of $21-\mathrm{cm}$ emission surveys.

The FUSE data were retrieved from the Multimission Archive at the Space Telescope Science Institute (MAST) and reduced locally using CALFUSEv2. $4^{3}$. Raw exposures within a single FUSE observation were coad-

3 Detailed calibration information can be found at http://fuse.pha.jhu.edu/analysis/calfuse_intro.html 
ded by channel midway through the pipeline. For further information on the reduction method see Danforth et al. (2006). The FUSE data have spectral resolution $\sim 20 \mathrm{~km} \mathrm{~s}^{-1}$, while the STIS echelle (E140M) has a resolution of $\sim 7 \mathrm{~km} \mathrm{~s}^{-1}$. (The FUSE data were binned by three pixels, where FUSE resolution is typically 8-10 pixels.) Archival STIS/E140M echelle spectra were reduced locally by S. Penton and smoothed over three pixels to form the $7 \mathrm{~km} \mathrm{~s}^{-1}$ resolution element. The FUSE and STIS data were both normalized in $10 \AA$ segments centered on the rest wavelength of each absorption feature. The continuum regions were selected interactively and fitted using Legendre polynomials up to sixth order. The signal-to-noise ratio, $S / N$, was designated as a $1 \sigma$ deviation from the fitted continuum. However for the FUSE data, we oversampled the resolution element (15$20 \mathrm{~km} \mathrm{~s}^{-1}$ ) by three pixels. The actual signal-to-noise is then $(S / N)_{\text {res }}=\sqrt{3}(S / N)_{\text {pix }}$. The STIS data were smoothed to the instrumental resolution prior to normalization, and the velocity was converted to the LSR for consistency.

In the STIS band, we examined five absorption lines: Si II $\lambda 1304.370$, Si II $\lambda 1526.707$, Si III $\lambda 1206.500$, and the Si IV doublet, $\lambda \lambda 1393.755,1402.770$. We did not include several other Si II lines $(\lambda 1190.4,1193.3,1260.4)$, because they were blended with other interstellar features or too saturated to be of value. However, Si II $\lambda 1020.699$ lies in the FUSE band, and in some cases produced a cleaner absorption feature. As a weaker line, Si II $\lambda 1020$ often provided a better velocity constraint than the saturated Si II lines seen with STIS.

The color coding in Figure 1 illustrates the partial segregation of redshifts and blueshifts on either side of the Galactic rotation axis passing through $\ell=180^{\circ}$. This effect has been seen in other species (Collins et al. 2005; Fox et al. 2006). Because HVC velocities are plotted in the LSR, the observed velocity segregation could be the result of the falloff in circular rotation velocity with elevation above the Galactic plane. In defining the LSR velocity for high-latitude HVCs, based on the Galactic rotation curve in the plane, we may have subtracted too large a circular velocity for directions at $\ell<180^{\circ}$, and the reverse at $\ell>180^{\circ}$. The result would be an apparent asymmetry in LSR velocities across the $\ell=180^{\circ}$ axis, as seen generally in Fig. 1. Although most previous HVC models assume that the circular velocity remains constant on cylinders, as one moves above the Galactic plane, a velocity lag has recently been noted in the Sloan Digital Sky Survey of $\sim 200,000$ F and G stars (Ivezić et al. 2008). A circular velocity lag in halo rotation has also been seen in external spiral galaxies (Collins, Benjamin, \& Rand 2002).

\section{DATA ANALYSIS}

\subsection{Reduction and Analysis of Data}

Once the data were normalized and placed on a common velocity scale, we stacked plots of the H I, Si II, Si III, and Si IV lines to observe whether the absorption features in each sight line share a velocity range. A variety of examples, involving both IVCs and HVCs, are shown in Figure 3. Although we detected Si II and Si IV in several lines, we selected one line with the cleanest data to derive the column density. We set the velocity ranges by hand in order to minimize the errors caused by weak or saturated absorption features. To define the velocity ranges, we typically found Si III $\lambda 1206.50$ to be the most useful, since it was always present and often quite strong. We used Si II and Si IV to identify velocity components and define the minimum velocity range, since their features, while harder to detect, did not have outer wings seen in the saturated Si III line. The $21 \mathrm{~cm}$ line provided useful velocity limits, especially when Si II and Si IV features were not prominent or did not agree.

All 37 sight lines exhibited either an IVC or HVC absorption feature. In 7 sight lines, we detected no HVCs: 3C 249, NGC 4051, NGC 4151, 3C 273, NGC 5548, Mrk 1383, and IR 2121-1757. These statistics suggest an HVC covering factor of $81 \pm 5 \%$ (30 out of 37 sight lines) of the high-latitude Galactic sky in Si III, with an error bar based on an uncertainty of \pm 2 sight lines in which we searched for Si III. This Si III covering fraction is higher than the $\sim 60 \%$ seen in O VI (Sembach et al. 2003), as expected from the relative strengths of Si III/O VI. Similar covering fractions of 80-90\% were found in our larger survey of Si III (Collins, Shull, \& Giroux 2009), in which 53 of 58 sight lines exhibited HVCs in Si III. In this paper, 8 of 37 sight lines exhibited three or more HVC/IVC components: Mrk 335, NGC 3783, PG 0953+415, 3C 351, Mrk 509, PHL 1811, UGC 12163, and NGC 7469. In these and other multiplecomponent cases, we treated the HVCs and IVCs as individual absorbers. However, in compiling statistics on the integrated column density of Si III, we combined the multiple HVC components into a single value per sight line.

For each absorption feature, we measured the equivalent width and the column density in order to compare the observational data to the theoretical models (Table 3 ). The column density, $N_{\mathrm{AOD}}$, for each absorption feature was found using the apparent optical depth (AOD) method described in Savage \& Sembach (1991). Our software takes into account two sources of error: continuum placement and velocity limits of integration. First, we moved the continuum up and down from the nominal placement by an amount equal to the the standard deviation of pixel values in the line-free regions, a relative change of $(S / N)^{-1}$. The AOD measurements using these upper and lower continua generate upper and lower bounds for $N_{\mathrm{AOD}}$. Second, we moved the limits of integration inward and outward from their nominal positions by $15 \mathrm{~km} \mathrm{~s}^{-1}$ and measured the column density for a second set of upper and lower bounds. For isolated lines, this resulted in negligible changes in $N_{\mathrm{AOD}}$, but for blended lines the difference can be significant. Since the two effects are uncorrelated, we subtract the nominal $N_{\text {AOD }}$ from the bounds and add the two upper and two lower uncertainties in quadrature for a final uncertainty.

The equivalent width, $W_{\lambda}$, is related to the column density, but it was most important for finding the cases where the the column density is an upper limit. The error in average equivalent width error at $\pm 1 \sigma$ and the average integration range error at the contracted and expanded velocity range were added in quadrature. In setting upper limits on column density, we adopted $4 \sigma$ as a 

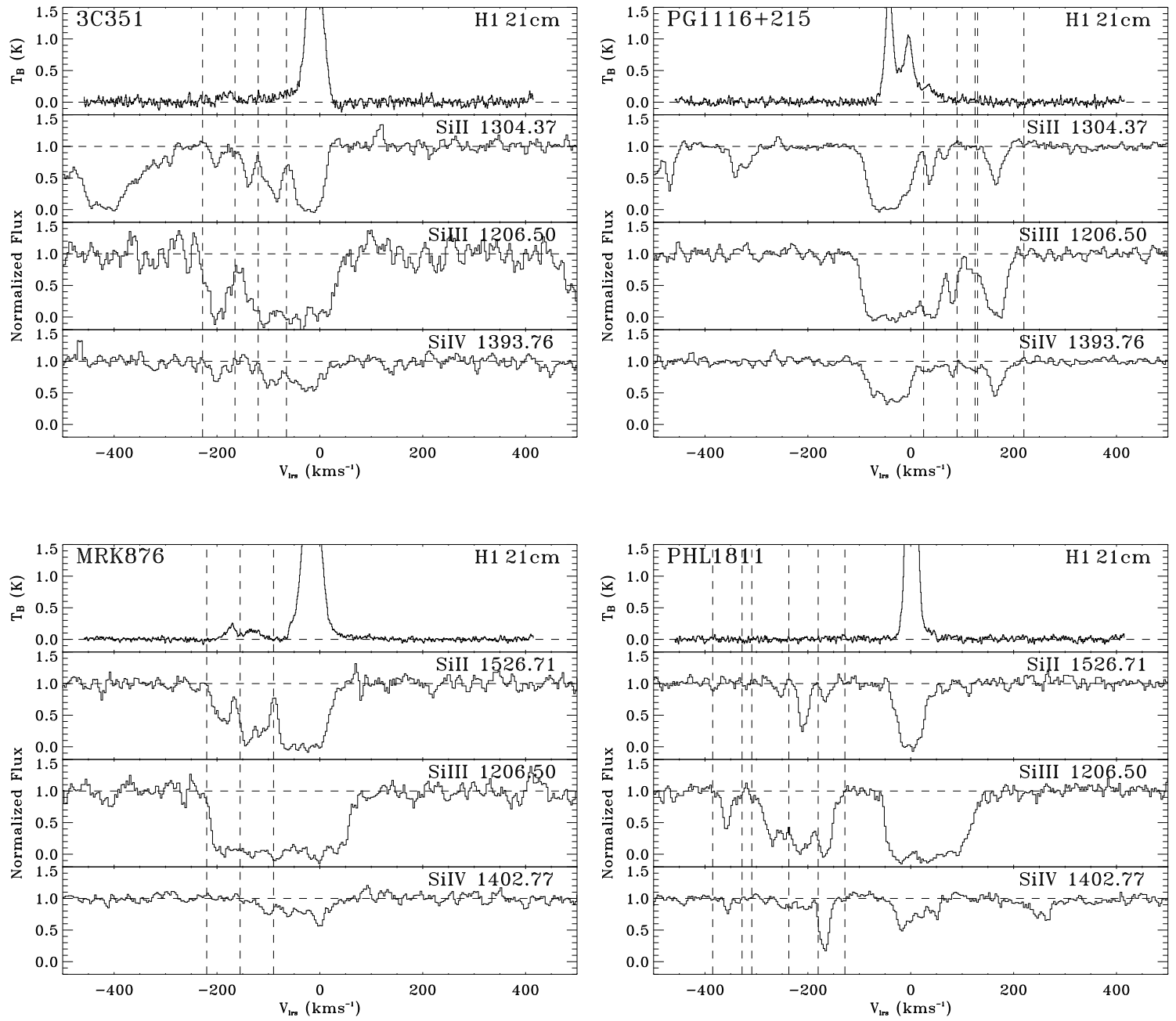

FIG. 3.- Normalized plots of HST/STIS spectra of 3C 351, PG 1116+215, Mrk 876, and PHL 1811, showing Si II, Si III, and Si IV absorption features and normalized plot of $\mathrm{H}$ I emission feature from the LDS. We mark with vertical dashed lines the LSR velocity range used to calculate the column densities.

conservative standard and used the relation,

$$
W_{\min }=\frac{4 \lambda_{0}}{R(S / N)} \text {. }
$$

Here, the instrumental resolution is $R=\lambda / \Delta \lambda$, which we take to be $R=15,000$ (FUSE) and $R=42,000$ (STIS/E140M). In the cases where $W_{\min }$ was less than the measured equivalent width, we assume that the column density has only a $4 \sigma$ significance.

The H I column density was measured by integrating the 21-cm spin temperature over the velocity range $v_{1} \leq$ $v \leq v_{2}$ (in $\mathrm{km} \mathrm{s}^{-1}$ ), using the formula,

$$
N_{\mathrm{HI}}=\left(1.823 \times 10^{18} \mathrm{~cm}^{-2}\right) \int_{v_{1}}^{v_{2}} T(v) d v,
$$

where the factor $1.823 \times 10^{18}$ converts from velocityintegrated temperature (in $\mathrm{K} \mathrm{km} \mathrm{s}^{-1}$ ) to H I column density (in $\mathrm{cm}^{-2}$ ). The errors in $N_{\mathrm{HI}}$ are typically smaller than those for the Si ion column densities and were calculated by the formula,

$\sigma_{\mathrm{NHI}}=\left(1.823 \times 10^{18} \mathrm{~cm}^{-2}\right)\left(v_{2}-v_{1}\right) \sqrt{\frac{\sum\left(T-T_{\text {mean }}\right)^{2}}{n-1}}$,

where $T(\mathrm{~K})$ is the spectral noise temperature, $\left(v_{2}-v_{1}\right)$ is the velocity range $\left(\mathrm{km} \mathrm{s}^{-1}\right)$, and $n$ is the number of temperature elements over that velocity range. We have not included uncertainty in $N_{\mathrm{HI}}$ from a mismatch to the LDS beam-size. Such effects can be important in some HVCs such as Complex C, with known sub-substructure, and have been estimated at $\pm 0.1 \operatorname{dex}$ in $\log \mathrm{N}_{\mathrm{HI}}$ (Wakker et al. 2003; Collins et al. 2007).

\subsection{Integrated Column Densities of Ionized Gas}

The high ions of heavy elements (C III, C IV, N V, $\mathrm{Si}$ III, Si IV, O VI) can be used as proxies for the amount of ionized hydrogen in the low halo. For ex- 
ample, the high-velocity O VI absorbers (Sembach et al. 2003) were found to have substantial mean column density, $\left\langle\log N_{\text {OVI }}\right\rangle=13.95 \pm 0.34$. Scaling from a fiducial metallicity of $0.2 Z_{\odot}$, we can estimate the corresponding ionized hydrogen column density,

$$
\begin{aligned}
\mathrm{N}_{\mathrm{HII}} & \approx \frac{\mathrm{N}_{\text {OVI }}}{(\mathrm{O} / \mathrm{H})_{\odot} f_{\mathrm{OVI}}\left(Z / Z_{\odot}\right)} \\
& \approx\left(4.5 \times 10^{18} \mathrm{~cm}^{-2}\right)\left(Z / 0.2 Z_{\odot}\right)^{-1} .
\end{aligned}
$$

Here, we adopt $(\mathrm{O} / \mathrm{H})_{\odot}=4.9 \times 10^{-4}$ as the solar oxygen abundance, assume that $f_{\text {OVI }} \approx 0.2$ is the O VI ionization fraction in hot gas at $\log T_{\max }=5.45$ (Sutherland \& Dopita 1993), and define $Z / Z_{\odot}$ as the oxygen metallicity relative to solar abundances. This high-velocity gas is likely to have metallicity in the range $Z_{\mathrm{HVC}}=0.1-$ $0.3 Z_{\odot}$, similar to that of the Magellanic Stream (Lu, Savage, \& Sembach 1994; Gibson et al. 2000; Sembach et al. 2001) or Complex C (Wakker et al. 1999; Gibson et al. 2001; Richter et al. 2001; Tripp et al. 2003; Collins, Shull, \& Giroux 2003, 2007).

A more recent survey (Fox et al. 2006) of O VI and C III examined 47 highly ionized HVCs (without 21-cm emission) at $100 \mathrm{~km} \mathrm{~s}^{-1}<\left|v_{\mathrm{VSR}}\right|<400 \mathrm{~km} \mathrm{~s}^{-1}$. This HVC population had mean column density $\left\langle\mathrm{N}_{\mathrm{OVI}}\right\rangle=$ $13.83 \pm 0.36$ and mean line width $\left\langle b_{\text {OVI }}\right\rangle=38 \pm$ $10 \mathrm{~km} \mathrm{~s}^{-1}$. In $81 \%$ (30 of 37) highly ionized HVCs, they found clear accompanying $\mathrm{C}$ III absorption with a ratio, $\mathrm{N}(\mathrm{C}$ III $) / \mathrm{N}(\mathrm{O} \mathrm{VI})$, which they claim is consistent with relative solar abundances and collisional ionization equilibrium at $T=1.7 \times 10^{5} \mathrm{~K}$. However, owing to the strong radiative cooling at these temperatures, the plasma is likely to be out of ionization equilibrium, which would alter the expected ratios.

The high-latitude ionized layer probed by the HVCs appears to have a total hydrogen column density $\sim$ $10^{19} \mathrm{~cm}^{-2}$, integrated through both sides of the Galactic plane. Depending on its spatial distribution and distance above the plane, this gas could provide a significant reservoir of low-metallicity material, some of which may cool and fall onto the Galactic disk. The total mass depends on the HVC distances and geometry. If the vertical column density, $N_{\mathrm{HII}}$, in these HVCs resides in a thin layer, above and below the Galactic disk with radius $R \approx(10 \mathrm{kpc}) R_{10}$, the total ionized mass is

$$
\begin{aligned}
M_{\mathrm{HII}} & \approx\left(2 \pi R^{2}\right)\left(1.32 m_{H}\right) N_{\mathrm{HII}} \\
& \approx\left(3 \times 10^{7} M_{\odot}\right) R_{10}^{2}\left(\frac{Z}{0.2 Z_{\odot}}\right)^{-1} .
\end{aligned}
$$

An HVC population around the Milky Way extending above a more extended disk $(R \approx 15 \mathrm{kpc})$ or with metallicity $\sim 0.1 Z_{\odot}$ could have mass exceeding $10^{8} M_{\odot}$.

¿From the Si III measurements, we now make the same calculation for the total hydrogen column density. As shown in Table 2, we detected Si III in 83 absorption systems: 61 HVCs along 30 sight lines and 22 IVCs along 20 sight lines. Because the Si III line is saturated in several cases, we list lower limits on the column density, $\log \mathrm{N}_{\text {SiIII }}$, computed by the AOD method. In order to compare Si III to O VI, we combine all HVCs in each sight line into a single Si III column density. Averaging the total N(Si III) along each sight line using measured values or lower limits, we find $\left\langle\log N_{\text {SiIII }}\right\rangle=13.42 \pm 0.21$ (for 30 sight lines with Si III HVCs) and $13.59 \pm 0.25$ (for 20 sightlines with 22 IVCs). We derive the total hydrogen mass by correcting for the (solar) silicon abundance, $(\mathrm{Si} / \mathrm{H})_{\odot}=3.24 \times 10^{-5}$, with a typical $\mathrm{HVC}$ metallicity $Z_{\mathrm{Si}} / Z_{\odot} \approx 0.2$ (values of $10-20 \%$ solar are observed in Complex C). We adopt an ionization fraction $f_{\text {SiIII }} \approx 0.7 \pm 0.2$ characteristic of multiphase conditions. We estimate that the HVC population corresponds to a vertical (one-sided) column density of ionized hydrogen,

$$
\begin{aligned}
\mathrm{N}_{\mathrm{HII}} & \approx \frac{\mathrm{N}_{\mathrm{SiIII}}}{(\mathrm{Si} / \mathrm{H})_{\odot} f_{\mathrm{SiIII}}\left(Z_{\mathrm{Si}} / Z_{\odot}\right)} \\
& \approx\left(6 \times 10^{18} \mathrm{~cm}^{-2}\right)\left(\frac{Z_{\mathrm{Si}}}{0.2 Z_{\odot}}\right)^{-1}\left(\frac{f_{\mathrm{SiII}}}{0.7}\right)^{-1}
\end{aligned}
$$

Perhaps coincidentally, this column density is close to that derived from O VI (eq. [5]), with mean column density $\left\langle\log \mathrm{N}_{\text {OVI }}\right\rangle=13.95 \pm 0.34$ (Sembach et al. 2003), although these ions probably trace gas in different thermal phases.

As noted by Collins et al. (2007), the hot gas at 10 $20 \%$ metallicity can cool on Gyr timescales, and the lowmetallicity $\mathrm{O}$ VI and $\mathrm{O}$ VII reservoir might produce a mass inflow rate of at least $0.1 M_{\odot} \mathrm{yr}^{-1}$. The Si III HVC absorbers observed in this survey probably have temperatures of $10^{4.0} \mathrm{~K}$ to $10^{4.5} \mathrm{~K}$. A rough estimate of the mass infall rate comes by dividing the mass of ionized gas (scaled from Si III HVCs) by the time for HVCs to fall from $10 \mathrm{kpc}$ to the plane at $100-300 \mathrm{~km} \mathrm{~s}^{-1}$. This estimate can also be corrected for H I using the typical ratio, 1.5-2.5, of total ( $\mathrm{H} \mathrm{I}+\mathrm{H}$ II) to ionized hydrogen (Collins et al. 2003) and by a factor of 0.5 for the fraction of HVCs falling inward. Averaged over the Galactic disk of radius $R=10-15 \mathrm{kpc}$, the population of Si III HVCs has a total hydrogen mass $\sim 10^{8} M_{\odot}$. Dividing by typical infall times of 30-100 Myr, we estimate an average mass infall rate $\sim 1 M_{\odot} \mathrm{yr}^{-1}$. Although uncertain, this is a substantial fraction of the replenishment rate needed to balance gas consumption by star formation in the disk (Gilmore 2001).

\subsection{Ionization Modeling}

Both HVCs and IVCs likely include multiphase gas, with a range of ionization mechanisms. Here, we examine the case of pure photoionization. For a subset of absorbers with the best data, including Si II, Si III, and Si IV, totaling $17 \mathrm{HVCs}$ and 19 IVCs, we were able to model the ionization conditions responsible for the silicon ions. In order to constrain the values of ionization parameter and metallicity, we compared the observations to a grid of models calculated using Cloudy v96.02 last described by Ferland et al. (1998). We assumed a planeparallel geometry, with no dust, trace elements, or molecular hydrogen. We set the lifetime of the HVC/IVC equal to $10^{8} \mathrm{yr}$, much longer than equilibrium timescales, and we ignored the effects of cosmic rays.

The most important parameter in our photoionization models is the photoionization parameter, $U=n_{\gamma} / n_{H}$, where $n_{\gamma}$ is the number density of ionizing photons and $n_{H}$ is the number density of hydrogen in all forms. For both AGN and OB-association sources, we assumed an ionizing radiation field parameterized by specific inten- 


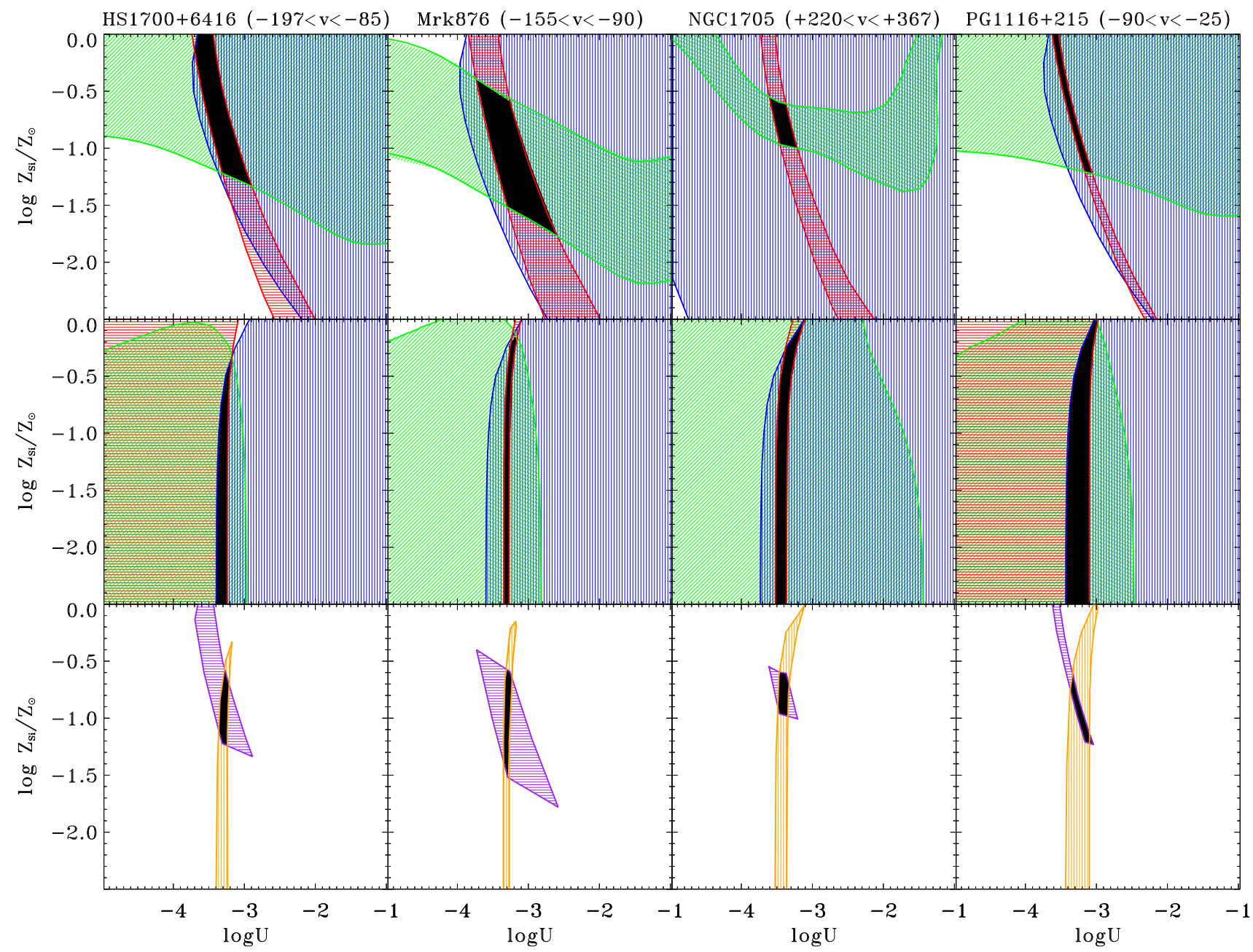

FIG. 4. - Photoionization models of the column densities of three Si ions constrain the ionization parameter, log $U$, and silicon metallicity, $Z_{\mathrm{Si}}$, for three HVCs (toward HS 1700+6417, Mrk 876, NGC 1705) and one IVC toward PG 1116+215. The color scheme in these panels shows regions constrained by the individual $\mathrm{Si}$ ion ratios and illustrate where model ion ratios match $(1 \sigma)$ observed ratios. In the top plots, we use $\log \left(N_{\mathrm{SiII}} / N_{\mathrm{HI}}\right), \log \left(N_{\mathrm{SiIII}} / N_{\mathrm{HI}}\right)$, and $\log \left(N_{\mathrm{SiIV}} / N_{\mathrm{HI}}\right)$. In middle plots, we show $\log \left(N_{\mathrm{SiIII}} / N_{\mathrm{SiIV}}\right), \log \left(N_{\mathrm{SiIII}} / N_{\mathrm{SiII}}\right)$, and $\log \left(N_{\mathrm{SiII}} / N_{\mathrm{SiIV}}\right)$. The bottom plots compare solutions from the top and middle plots. Black regions show overlapping solutions.

sity $J_{\nu}=J_{0}\left(\nu / \nu_{0}\right)^{-\alpha}$ in units $\mathrm{erg} \mathrm{cm}^{-2} \mathrm{~s}^{-1} \mathrm{~Hz}^{-1} \mathrm{sr}^{-1}$. For metagalactic radiation, the specific intensity at 1 ryd (Shull et al. 1999) lies in the range $J_{0} \approx(1-2) \times 10^{-23}$, which corresponds to a normally incident (one-sided) photon flux of $\Phi_{0} \approx 10^{4} \mathrm{~cm}^{-2} \mathrm{~s}^{-1}$. The mean spectral index is chosen as $\langle\alpha\rangle=1.5$, consistent with the observed spectral indices (rest-frame 1-3 ryd) for AGN at redshifts $z \geq 0.3$ (Telfer et al. 2002) modified by transmission and reprocessing through the IGM (Fardal et al. 1998). The shape of metagalactic ionizing spectrum is still uncertain, as indicated by the fact that composite spectra of AGN at $z<0.3$ observed by FUSE appear to have somewhat harder spectra with $\langle\alpha\rangle=0.5-0.6$ (Scott et al. 2004; Shull et al. 2009).

For HVCs within the Galactic halo, the radiation field is expected to be incident primarily from one side, dominated by OB-star radiation escaping from the Galactic disk. This Galactic ionizing radiation field is estimated to be 10-100 times stronger than the metagalactic field (Giroux \& Shull 1997). We scale the normally incident flux of hydrogen-ionizing photons to the value, $\Phi_{0}=\left(10^{5.5}\right.$ photons $\left.\mathrm{cm}^{-2} \mathrm{~s}^{-1}\right) \Phi_{5.5}$, typical of gas in the low Galactic halo (Dove \& Shull 1994; Bland-Hawthorne \& Maloney 1999). Integrating over frequency and angle, we can relate $J_{0}$ to the number density, $n_{\gamma}$, of ionizing photons, the one-sided photon flux $\Phi_{0}$, and the photoionization parameter, $U=n_{\gamma} / n_{H}$ :

$$
\begin{aligned}
\Phi_{0} & =\left(\frac{2 \pi J_{0}}{h c \alpha}\right)=\left(10^{5.5} \text { photons } \mathrm{cm}^{-2} \mathrm{~s}^{-1}\right) \Phi_{5.5}, \\
n_{\gamma} & =\frac{\Phi_{0}}{c}=\left(1.05 \times 10^{-5} \mathrm{~cm}^{-3}\right) \Phi_{5.5}\left(\frac{1.5}{\alpha}\right), \\
U & =\frac{n_{\gamma}}{n_{H}}=\left(1.05 \times 10^{-3}\right) \Phi_{5.5} n_{-2}^{-1}\left(\frac{1.5}{\alpha}\right) .
\end{aligned}
$$

Here, we scaled the HVC hydrogen density to typical values, $n_{H}=\left(10^{-2} \mathrm{~cm}^{-3}\right) n_{-2}$. For instance, our photoionization models of multiple ion stages in Complex $\mathrm{C}$ (Gibson et al. 2001; Collins et al. 2003, 2007) find $n_{H} \approx$ $0.01-0.1 \mathrm{~cm}^{-3}$.

For a normally incident radiation field, the photon flux is $\Phi_{0}=n_{\gamma} c$, while an isotropic radiation field gives $\Phi_{0}=n_{\gamma} c / 4$. Thus, to relate photoionization parameter 

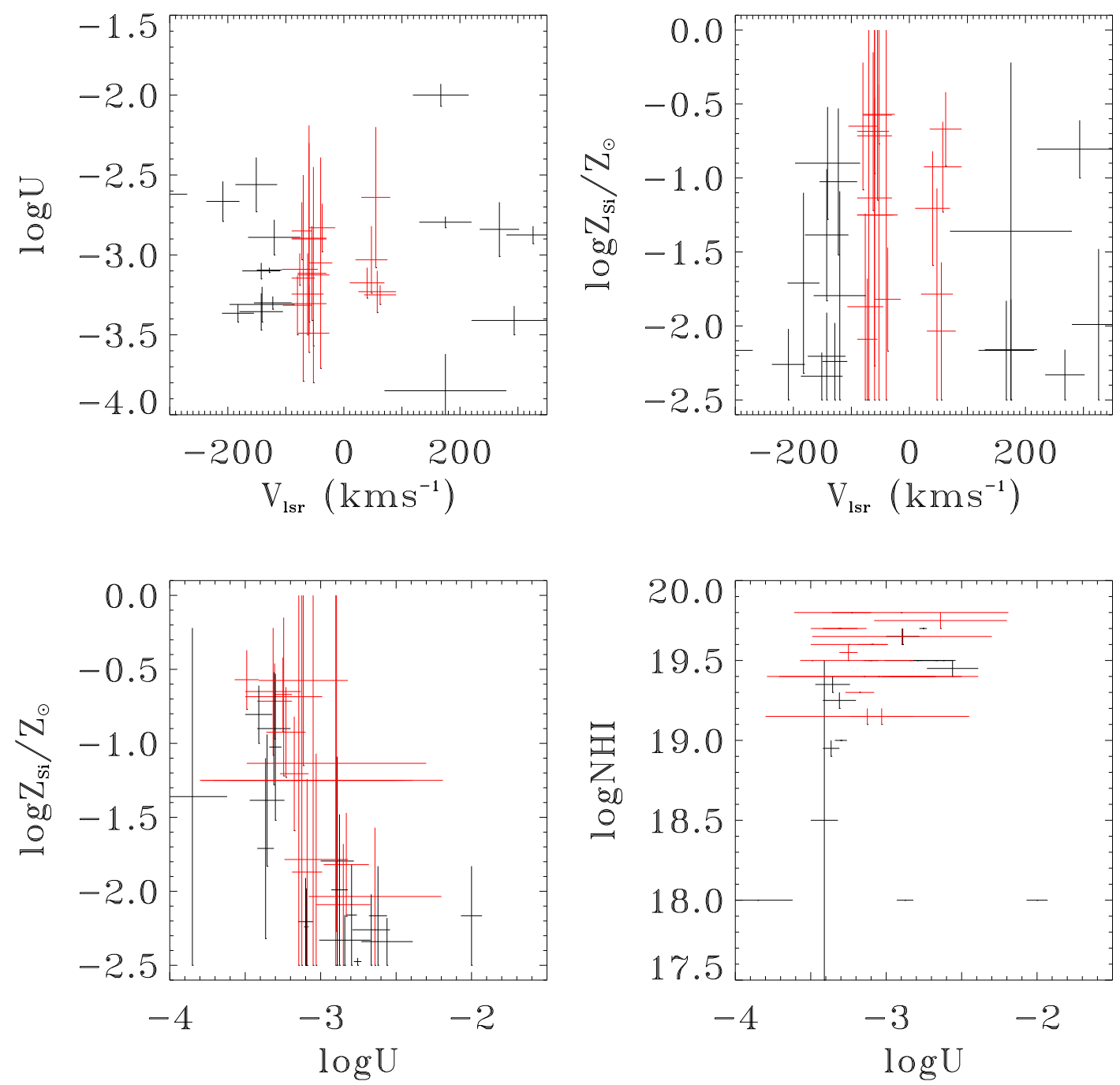

FIG. 5. - Correlation between different model parameters $\left(U, Z_{\mathrm{Si}}\right)$ and absorber LSR velocity $\left(v_{\mathrm{LSR}}\right)$ and column density $\left(\log N_{\mathrm{HI}}\right)$. Red points show models for IVCs, and black are for HVCs. We see that $\log U$ is fairly constant, with little dependence on $N_{\mathrm{HI}}, Z_{\mathrm{Si}}$, or $v_{\mathrm{LSR}}$. We see an apparent tilt in the relation between $Z_{\mathrm{Si}}$ and $\log U$, which may arise from higher densities in IVCs compared to HVCs.

$U$ to $n_{\gamma}$, one must specify the geometry of the radiation field (one-sided normally-incident or diffuse isotropic). We explored a range $-4<\log U<-1$ and constructed a three-dimensional grid of models with parameters: total hydrogen density $n_{H}$, neutral hydrogen column density $N_{\mathrm{HI}}$, and silicon metallicity $Z_{\mathrm{Si}} / Z_{\odot}$. We varied the metallicity over the range $-2.5<\log \left(Z_{\mathrm{Si}} / Z_{\odot}\right)<0$ and the $\mathrm{H} \mathrm{I}$ column density over the range $17<\log$ $N_{\mathrm{HI}}\left(\mathrm{cm}^{-2}\right)<20$ in logarithmic steps of 0.1 dex. We varied the hydrogen density from $10^{-4}<n_{H}\left(\mathrm{~cm}^{-3}\right)<1$, spanning a broad range in density from diffuse intergalactic medium (IGM) to diffuse interstellar medium (ISM). Each model produced column densities for many species, including Si II, Si III, Si IV, which are studied relative to $\mathrm{H}$ I. We then compared model column density ratios to the observed ratios for all absorbers (HVCs and IVCs). From the observed $N_{\mathrm{HI}}$, we then found solutions for $n_{H}$ and $Z_{\mathrm{Si}} / Z_{\odot}$. The quoted error bars reflect the range in parameters $\left(U\right.$ and $Z_{\mathrm{Si}}$ ) allowed by the photoionization models. We have not included systematic errors arising from assumptions about the spectral shape of the photoionizing radiation field, or the relative contributions of collisional ionization from hot gas.

The question of how to properly model the ionization corrections for multiphase HVCs is complicated and beyond the scope of this paper. One possible approach to address this issue would use multiple ion states from another element (C II, C III, C IV) to constrain the ionization ratio (photoionized/collisional). Another approach is to use the "neutral-gas" ratios, [O I/H I], [S II/H I], $[\mathrm{Si} \mathrm{II} / \mathrm{H} \mathrm{I}]$, to derive the metallicity, and then assume that this metallicity applies to the higher ions, Si III, Si IV, C III, C IV. We will explore these approaches in a subsequent paper. For now, we simply note that the photoionization modeling done here introduces systematic uncertainties in the HVC (silicon) metallicities, by the lack of any correction for Si III and Si IV produced in hot, collisionally ionized gas. Thus, the low values of $Z_{\mathrm{Si}}$ described in $\S 4$ may reflect the possibility that Si III and Si IV are not co-spatial with the H I. 

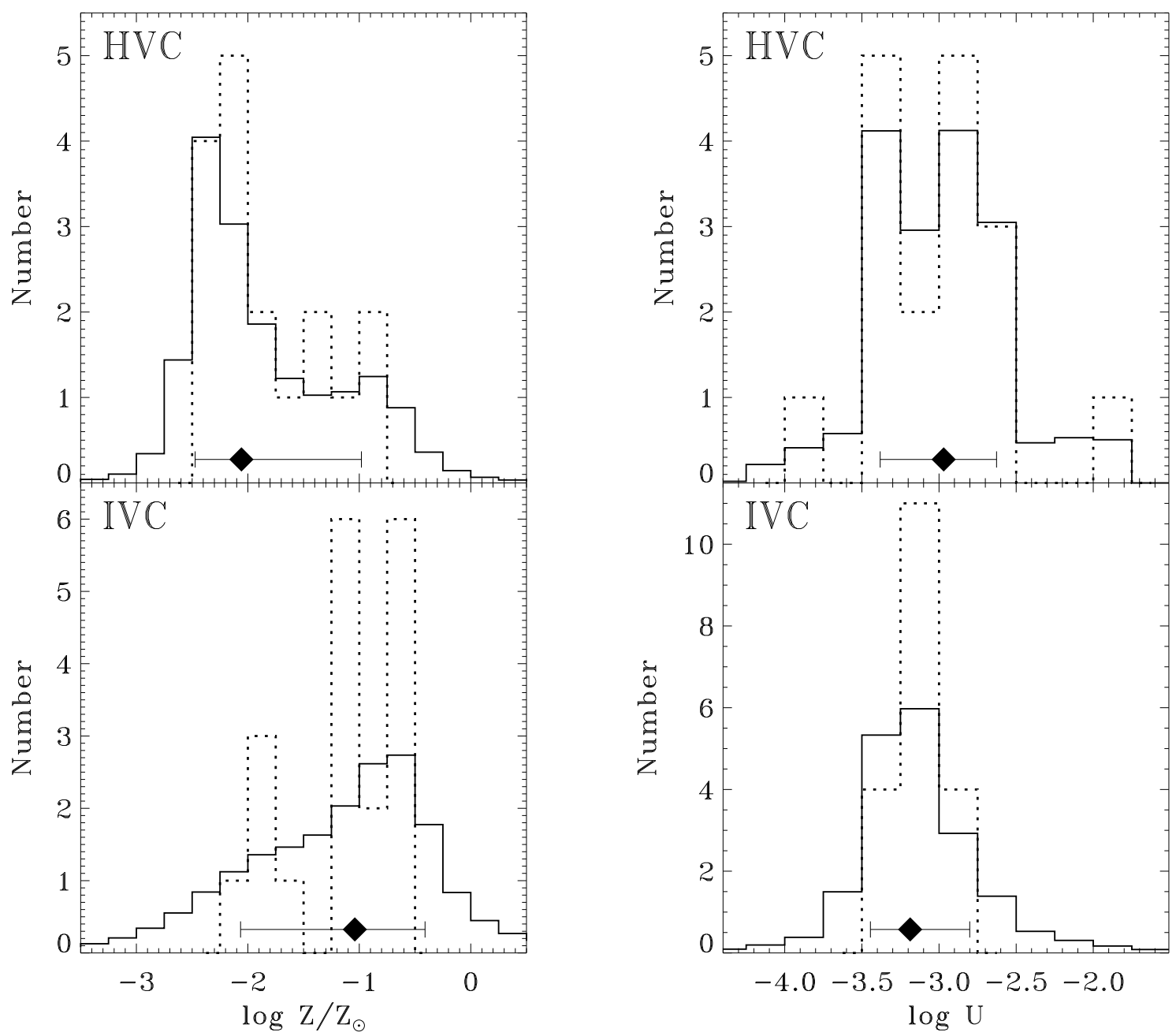

FIG. 6.- Histograms of $\log U$ and $\log \left(Z_{\mathrm{Si}} / Z_{\odot}\right)$ for 17 modeled HVCs (top panels) and 19 modeled IVCs (bottom panels), assuming pure photoionization. More reliable metallicities of 10-30\% solar can be derived from [Si II/H I]. Our multi-bin histogram technique (see text) accounts for the distribution of metallicities and ionization parameters into adjacent bins, assuming a Gaussian distribution. Diamonds show medians (with $1 \sigma$ variance in cumulative distribution). The median ionization parameter is well determined at $\log U=-3.0_{-0.4}^{+0.3}$ for HVCs, while metallicities are highly uncertain, with a broad range from $\log Z_{\mathrm{Si}} / Z_{\odot}=-2.5$ to 0 , with median $\left\langle\log \left(Z_{\mathrm{Si}} / Z_{\odot}\right)\right\rangle=-2.06_{-0.41}^{+1.08}$ for 17 modeled HVC, and $-1.04_{-1.03}^{+0.63}$ for 19 modeled IVCs.

\section{RESULTS AND DISCUSSION}

The column density ratios of the three silicon ions relative to $N_{\mathrm{HI}}$ were compared to photoionization models to match the HVC/IVC characteristics. The ionization parameter, $\log U$, is best constrained by the pairs of individual silicon ions, Si II, Si III, and Si IV. Once we have a solution for $\log U$, we constrain the Si metallicity by the ratio of silicon column densities to $\log N_{\mathrm{HI}}$. The Si III did not provide as much of a constraint on the model ratios as the weaker Si II and Si IV ions. However, because of its strength and sensitivity, Si III was used to identify HVCs and define their velocity ranges. These models yield a range of silicon metallicity $\left(Z_{\mathrm{Si}}\right)$ and hydrogen density $\left(n_{H}\right)$. Because $U \propto \Phi_{H} / n_{H}$, we can then infer the photoionizing flux, $\Phi_{H}$. From $U$ and $Z_{\mathrm{Si}}$, we can estimate the location of the HVCs. Sample solutions for $U$ and $Z_{\mathrm{Si}}$ in four absorbers (three HVCs and one IVC) are displayed in Figure 4, which illustrates our method for determining $\log U$ and $\log Z_{\mathrm{Si}}$.

The full solutions are given in Table 3, and Figure 5 displays the correlations among metallicity, ionization parameter, velocity, and $N_{\mathrm{HI}}$. We observe an apparent tilt in the relation between $Z_{\mathrm{Si}}$ and $\log U$, which may arise from higher densities in IVCs compared to HVCs. Figure 6 presents a set of histograms that show the range of ionization parameter $U$ and metallicity $Z_{\mathrm{Si}} / Z_{\odot}$. Because we can determine $U$ more accurately than $Z_{\mathrm{Si}}$, we have plotted these quantities using a multi-bin histogram technique that distributes power into adjacent bins assuming a Gaussian distribution; see Danforth \& Shull (2008) for more details.

The models for $\mathrm{Si}$ metallicity spanned the range $-2.5 \leq \log \left(Z_{\mathrm{Si}} / Z_{\odot}\right) \leq 0$, with mean values $\left\langle\log \left(Z_{\mathrm{Si}} / Z_{\odot}\right)\right\rangle=-2.1_{-0.4}^{+1.1}$ (for 17 modeled HVCs) and $-1.0_{-1.0}^{+0.6}$ (for 19 modeled IVCs). These metallicities are somewhat lower than the mean (column-density 
weighted) value, $\left\langle\log \left(Z_{\mathrm{O}} / Z_{\odot}\right)\right\rangle=-0.89$ found for Complex C (Collins et al. 2007). They are also lower than the metallicities of "neutral gas" estimated from [Si II/H I] and of the LMC and SMC. However, they agree fairly well with the mean metallicities, $[\mathrm{Fe} / \mathrm{H}]=-1.46 \pm 0.30$ measured for $\sim 200,000$ halo F/G stars in the Sloan Digital Sky Survey (Ivezić et al. 2008).

The range of metallicities in IVCs extends to higher values than in the HVCs. In the lowest-column bins of IVCs, three of the four absorbers could be a part of a larger HVC system. This distinction may not be significant, owing to the definition used to separate HVCs and IVCs at $90-100 \mathrm{~km} \mathrm{~s}^{-1}$. Within the set of models, the ionization parameter ranged from $-4 \leq \log U \leq-1$. The solutions for the $61 \mathrm{HVCs}$ and 22 IVCs showed that most could be explained by $\log U \approx-3.0 \pm 0.3$. Similar modeling for absorbers in the low-redshift IGM (Danforth \& Shull 2005, 2008) found $\log U \approx-2.1 \pm 0.5$, for a diffuse, isotropic radiation field. Thus, the HVCs appear to have an ionization parameter ten times lower than that of the IGM, suggesting that HVCs reside in the higher-pressure Galactic halo, with a larger $n_{H}$.

Whether the HVC metallicity differences between the silicon-ion photoionization models and $[\mathrm{O} \mathrm{I} / \mathrm{H} \mathrm{I}]$ are statistically significant may depend on systematic uncertainties in our photoionization modeling. These models are based on ratios of Si II, Si III, and Si IV column densities (from UV absorption lines) to $N_{\mathrm{HI}}$ determined from 21-cm emission. Beam-size effects could make a difference in matching the $\mathrm{H}$ I to the absorbing sight line. We believe the mean ionization parameter to be fairly accurately determined at $\log U \approx-3.0 \pm 0.3$, but the range of metallicities is somewhat larger. The typical uncertainties for individual sight lines are \pm 0.1 in $\log U$ and \pm 0.5 in metallicity $\log Z_{\mathrm{Si}}$.

We now summarize the main results of our survey.

1. The strong Si III $1206.50 \AA$ absorption line is a sensitive tracer of high-velocity and intermediatevelocity ionized gas, typically with $4-5$ times higher optical depth than O VI $\lambda 1031.93$. With highresolution UV spectrographs, this allows sensitivity to HVCs with $N_{\text {SiIII }}$ as low as $5 \times 10^{11} \mathrm{~cm}^{-2}$, corresponding to ionized hydrogen column densities $N_{H} \approx 8 \times 10^{16} \mathrm{~cm}^{-2}$ at $0.2 Z_{\odot}$ metallicity.

2. The HVC covering factor of the high-latitude Galactic sky in Si III is $81 \pm 5 \%$, somewhat higher than seen in in O VI (60\%) but much larger than in H I 21-cm emission (37\%). The mean HVC column density per sight line is $\left\langle\log N_{\mathrm{SiIII}}\right\rangle=13.42 \pm 0.21$ for 30 sight lines with 61 HVCs and $13.59 \pm 0.25$ for 20 sight lines with 22 IVCs.

3. Correcting for silicon metallicity and ionization fraction, we estimate that the Si III corresponds to a column density of ionized hydrogen, $\mathrm{N}_{\mathrm{HII}} \approx$ $\left(6 \times 10^{18} \mathrm{~cm}^{-2}\right)\left(Z_{\mathrm{Si}} / 0.2 Z_{\odot}\right)^{-1}$, similar to that inferred from the population of O VI HVCs (Sembach et al. 2003). If this ionized HVC layer extends $\sim 10 \mathrm{kpc}$ above and below the Galactic plane, it could contain $10^{8} M_{\odot}$ of low-metallicity (10-20\% solar) gas. The infall times for HVCs at $10 \mathrm{kpc}$ and $100-200 \mathrm{~km} \mathrm{~s}^{-1}$ are $50-100 \mathrm{Myr}$. This infall averages $1 M_{\odot} \mathrm{yr}^{-1}$, a substantial portion of the replenishment rate for star formation in the disk.

4. Using all three silicon ions (Si II, Si III, Si IV) and assuming pure photoionization, we infer a mean photoionization parameter, $\log U \approx-3.0 \pm 0.2$, and average (highly uncertain) silicon metallicity, $\left\langle\log \left(Z_{\mathrm{Si}} / Z_{\odot}\right)\right\rangle=-1.4 \pm 0.3(-1.8 \pm 0.4$ for HVCs and $-1.2 \pm 0.4$ for IVCs). This ionizing radiation field is $\sim 10$ times lower than inferred in the low- $z$ IGM. The HVC metallicities inferred from [Si II/H I] are 10-30\%. The lower metallicities found from all three ions are unreliable, because of the likely contributions from collisional ionization.

5. We see HVC velocity segregation on either side of the rotation axis, with blue-shifted absorbers $\left(V_{\mathrm{LSR}}<-90 \mathrm{~km} \mathrm{~s}^{-1}\right)$ found primarily at $\ell<180^{\circ}$ and red-shifted gas $\left(V_{\mathrm{LSR}}>+90 \mathrm{~km} \mathrm{~s}^{-1}\right)$ at $\ell>$ $180^{\circ}$. This effect has been seen in other species (Collins et al. 2005; Fox et al. 2006) and may result from a lag in the circular rotation velocity at elevations off the disk plane.

In the future, we eagerly await the installation of the Cosmic Origins Spectrograph in Servicing Mission 4 for the Hubble Space Telescope. This mission may also repair STIS. Together, these UV spectrographs will rejuvenate spectroscopic studies of HVCs and IVCs towards many AGN targets. With COS, we have targeted ten sight lines toward HVCs in Complex C, Complex A, WD, WB, and several negative-velocity O VI HVCs. These studies, at $\mathrm{S} / \mathrm{N} \approx 30-40$ and $15 \mathrm{~km} \mathrm{~s}^{-1}$ resolution, will survey physical conditions and abundances in cool, warm, and hot HVC gas. Based on our experience in this paper, we believe that the silicon ions will play an important role in defining the ionization conditions and $\mathrm{HVC} / \mathrm{IVC}$ kinematics.

Our group's research support at the University of Colorado for UV studies of the IGM and Galactic halo gas comes from COS grant NNX08-AC14G and STScI spectroscopic archive grants (AR-10645.02-A and AR11773.01-A). We also have support from NSF grant AST07-07474. We thank Steve Penton and Jason Tumlinson for reducing the initial STIS and FUSE data and Gary Ferland and Mark Giroux for useful discussions regarding the CLOUDY modeling. We also thank the referee for insights and comments that improved our arguments. This work contains data obtained for the Guaranteed Time Team by the NASA-CNES-CSA FUSE mission operated by the Johns Hopkins University, as well as data from the Hubble Space Telescope. 


\section{REFERENCES}

Adams, W. S. 1949, ApJ, 109, 354

Asplund, M., Grevesse, N., \& Sauval, A. J. 2005, in Cosmic Abundances as Records of Stellar Evolution and Nucleosynthesis, ASP Conf. Ser. 336, 25

Braun, R., \& Burton, W. B. 1999, A\&A, 341, 437

Bland-Hawthorne, J., \& Maloney, P. 1999, ApJ, 510, L33

Blitz, L., Spergel, D. N., Teuben, P. J., Hartmann, D., \& Burton, W. B. 1999, ApJ, 514, 818

Bregman, J. N. 1980, ApJ, 236, 577

Bregman, J. N., \& Lloyd-Davies, E. J. 2007, ApJ, 669, 990

Collins, J. A., Benjamin, R. A., \& Rand, R. J. 2002, ApJ, 578,98

Collins, J. A., Shull, J. M., \& Giroux, M. L. 2003, ApJ, 585, 336

Collins, J. A., Shull, J. M., \& Giroux, M. L. 2004, ApJ, 605, 216

Collins, J. A., Shull, J. M., \& Giroux, M. L. 2005, ApJ, 623, 196

Collins, J. A., Shull, J. M., \& Giroux, M. L. 2007, ApJ, 657, 271

Collins, J. A., Shull, J. M., \& Giroux, M. L. 2009, ApJ, in preparation

Danforth, C. W., \& Shull, J. M. 2005, ApJ, 625, 555

Danforth, C. W., \& Shull, J. M. 2008, ApJ, 679, 194

Danforth, C. W., Shull, J. M., Rosenberg, J. L., \& Stocke, J. T. 2006, ApJ, 640, 716

Dove, J. B., \& Shull, J. M. 1994, ApJ, 430, 222

Erb, D. K., Shapley, A. E., Pettini, M., Steidel, C. C., Reddy, N. A., \& Adelberger, K. C. 2006, ApJ, 644, 813

Fang, T., et al. 2002, ApJ, 572, L127

Fardal, M. A., Giroux, M. L., \& Shull, J. M. 1998, AJ, 115, 2206

Ferland, G. J., Korista, K. T., Verner, D. A., Ferguson, J. W., Kingdon, J. B., \& Verner, E. M. 1998, PASP, 110, 761

Fox, A. J., Savage, B. D., \& Wakker, B. D. 2006, ApJS, 165, 229

Gibson, B. K., Giroux, M. L., Penton, S. V., Putman, M. E., Stocke, J. T., \& Shull, J. M. 2000, AJ, 120, 1830

Gibson, B. K., Giroux, M. L., Penton, S. V., Stocke, J. T., \& Shull, J. M., \& Tumlinson, J. 2001, AJ, 122, 3280

Gilmore, G. 2001, in Galaxy Disks \& Disk Galaxies, ASP Conf. Ser., 230, 3

Giroux, M. L., \& Shull, J. M. 1997, AJ, 113, 1505

Hartmann, D., \& Burton, W. B. 1997, Atlas of Galactic Neutral Hydrogen (Cambridge: Cambridge Univ. Press)

Hobbs, L. M. 1965, ApJ, 142, 160

Houck, J. C., \& Bregman, J. N. 1990, ApJ, 352, 506

Indebetouw, R., \& Shull, J. M. 2004, ApJ, 607, 309
Ivezić, Z., et al. 2008, ApJ, 684, 287

Lu, L., Savage, B. D., \& Sembach, K. R. 1994, ApJ, 437, L119

Maller, A. H., \& Bullock, J. S. 2004, MNRAS, 355, 694

McKernan, B., et al. 2004, ApJ, 617, 232

Morton, D. C. 2003, ApJS, 149, 205

Münch, G., \& Zirin, H. 1961, ApJ, 133, 11

Murphy, E. M., Lockman, F. J., \& Savage, B. D. 1995, ApJ, 447,642

Nicastro, F., et al. 2002, ApJ, 573, 157

Oort, J. H. 1966, B.A.N., 18, 421

Richter, P., et al. 2001, ApJ, 559, 318

Savage, B. D., \& Sembach, K. R. 1991, ApJ, 379, 245

Savage, B. D., et al. 2003, ApJS, 146, 125

Scott, J. E., Kriss, G. A., Brotherton, M., Green, R. F., Hutchings, J., Shull, J. M., \& Zheng, W. 2004, ApJ, 615, 135

Sembach, K. R., \& Savage, B. D. 1992, ApJS, 83, 147

Sembach, K. R., Savage, B. D., Lu, L., \& Murphy, E. M. 1995, ApJ, 451, 616

Sembach, K. R., et al. 1999, ApJ, 515, 108

Sembach, K. R., Howk, J. C., Savage, B. D., Shull, J. M., \& Oegerle, W. D. 2001, ApJ, 561, 573

Sembach, K. R., et al. 2003, ApJS, 146, 165

Sembach, K. R., et al. 2003, ApJS, 150, 387

Shapiro, P. R., \& Field, G. B. 1976, ApJ, 205, 762

Shull, J. M., \& Slavin, J. D. 1994, ApJ, 427, 784

Shull, J. M., Roberts, D., Giroux, M. L., Penton, S. V., \& Fardal, M. A. 1999, AJ, 118, 1450

Shull, J. M., Clausen, D., Danforth, C. W., \& Giroux, M. L. 2009, ApJ, to be submitted.

Spitzer, L. 1956, ApJ, 124, 20

Sutherland, R. S., \& Dopita, M. A. 1993, ApJS, 88, 253

Telfer, R., et al. 2002, ApJ, 565, 773

Thom, C., Peek, J. E. G., Putman, M. E., Heiles, C., Peek, K. M. G., \& Wilhelm, R. 2008, ApJ, 684, 364

Tripp, T. M., et al. 2003, AJ, 125, 3122

Tremonti, C. A., et al. 2004, ApJ, 613, 898

Tumlinson, J., et al. 2004, ApJ, 566, 857

Wakker, B. P., \& van Woerden, H. 1997, ARA\&A, 35, 217

Wakker, B. P., et al. 1999, Nature, 402, 388

Wakker, B. P., et al. 2007, ApJ, 670, L113

Wakker, B. P., et al. 2008, ApJ, 672, 298

Yao, Y., \& Wang, Q. D. 2007, ApJ, 658, 1088

Yao, Y., Nowak, M. A., Wang, Q. D., Schulz, N. S., \&

Canizares, C. R. 2008, ApJ, 672, L21 
TABLE 2

HVC AND IVC Sight-Line ${ }^{a}$ Statistics

\begin{tabular}{|c|c|c|c|c|c|c|}
\hline Sightline & $\begin{array}{l}\text { Velocity Range } \\
\qquad\left(\mathrm{km} \mathrm{s}^{-1}\right)\end{array}$ & $\begin{array}{c}\text { Absorber } \\
\text { Type }\end{array}$ & $\begin{array}{c}\log N_{\mathrm{HI}} \\
\left(\mathrm{N} \text { in } \mathrm{cm}^{-2}\right)\end{array}$ & $\begin{array}{c}\log N_{\mathrm{SiII}} \\
\left(\mathrm{N} \text { in } \mathrm{cm}^{-2}\right)\end{array}$ & $\begin{array}{c}\log N_{\mathrm{SiIII}} \\
\left(\mathrm{N} \text { in } \mathrm{cm}^{-2}\right)\end{array}$ & $\begin{array}{c}\log N_{\mathrm{SiIV}} \\
\left(\mathrm{N} \text { in } \mathrm{cm}^{-2}\right)\end{array}$ \\
\hline \multirow[t]{5}{*}{ Mrk 335} & $-431,-385$ & HVC & $<19.37$ & $12.71_{-0.14}^{+0.15}$ & $12.41_{-0.06}^{+0.11}$ & $<12.25$ \\
\hline & $-365,-280$ & HVC & $<19.37$ & $14.24_{-0.28}^{+0.14}$ & $12.99_{-0.03}^{+0.05}$ & $<12.47$ \\
\hline & $-264,-238$ & HVC & $<19.37$ & $\begin{array}{l}<12.58 \\
-0.28\end{array}$ & $12.29_{-0.10}^{+0.03}$ & $12.47_{-0.12}^{+0.24}$ \\
\hline & $-137,-85$ & HVC & $<19.37$ & $12.96_{-0.13}^{+0.11}$ & $\geq 13.44$ & $<12.25$ \\
\hline & $-85,-25$ & IVC & $19.48_{-0.13}^{+0.10}$ & $14.74_{-0.12}^{+0.13}$ & $\geq 13.63$ & $12.97_{-0.10}^{+0.08}$ \\
\hline \multirow[t]{2}{*}{ Ton S210 } & $-251,-198$ & HVC & $<19.66$ & $<12.57$ & $12.88_{-0.09}^{+0.08}$ & $12.80_{-0.05}^{+0.08}$ \\
\hline & $-198,-127$ & HVC & $<19.66$ & $13.19_{-0.01}^{+0.08}$ & $\geq 13.68$ & $<12.47$ \\
\hline \multirow[t]{2}{*}{ HE $0226-440$} & 50,90 & IVC & $<18.00$ & $<12.59$ & $12.10_{-0.30}^{+0.11}$ & $<12.05$ \\
\hline & 119,215 & $\mathrm{HVC}$ & $<18.00$ & 12.35 & $13.18_{+0.05}^{+0.09}$ & $12.71 \pm 0.14$ \\
\hline \multirow[t]{2}{*}{ PKS 0312-770 } & 70,280 & HVC & $<18.00$ & $\geq 14.93$ & $\geq 14.32$ & $13.28_{-0.05}^{+0.07}$ \\
\hline & 280,372 & HVC & $<18.00$ & $13.29 \pm 0.06$ & $\overline{\geq} 13.61$ & $13.28 \pm 0.06$ \\
\hline PKS 0405-12 & 110,170 & $\mathrm{HVC}$ & $<19.52$ & $<12.55$ & $12.50 \pm 0.09$ & $12.64_{-0.16}^{+0.16}$ \\
\hline \multirow[t]{2}{*}{ NGC 1705} & 90,143 & $\mathrm{HVC}$ & $\geq 19.67$ & $\geq 14.63$ & $12.69_{-0.09}^{+0.13}$ & $<12.07$ \\
\hline & 210,357 & $\mathrm{HVC}$ & $17.48 \pm 0.20$ & $13.55 \pm 0.04$ & $\geq 13.67$ & $12.80_{-0.14}^{+0.16}$ \\
\hline \multirow[t]{3}{*}{ HS 0624+6907 } & $-150,-90$ & HVC & $19.19_{-1.00}^{+0.28}$ & $14.15_{-0.12}^{+0.14}$ & $13.13_{-0.20}^{+0.12}$ & $<12.54$ \\
\hline & $-90,-60$ & IVC & $<19.63$ & $\geq 13.91$ & $\geq 13.41$ & $12.71_{-0.25}^{+0.31}$ \\
\hline & 100,135 & HVC & $<19.63$ & $<12.43$ & $12.26_{-0.12}^{+0.23}$ & $<12.00$ \\
\hline \multirow[t]{4}{*}{ PG $0953+415$} & $-185,-145$ & HVC & $<19.32$ & $12.76_{-0.13}^{+0.46}$ & $12.41_{-0.16}^{+0.13}$ & $12.71_{-0.15}^{+0.24}$ \\
\hline & $-145,-100$ & HVC & $<19.32$ & $<12.30$ & $\geq 13.31$ & $12.66_{-0.14}^{+0.22}$ \\
\hline & 95,150 & HVC & $<19.32$ & $<12.30$ & $12.73_{-0.05}^{+0.08}$ & $<12.05$ \\
\hline & 150,185 & $\mathrm{HVC}$ & $<19.32$ & $<12.30$ & $11.92_{-0.28}^{+0.33}$ & $<12.40$ \\
\hline Ton 28 & 95,180 & $\mathrm{HVC}$ & $<19.53$ & $<12.70$ & $12.75 \pm 0.07$ & $12.92_{-0.12}^{+0.13}$ \\
\hline $3 \mathrm{C} 249$ & $-80,-30$ & IVC & $19.72_{-0.08}^{+0.07}$ & $\geq 14.61$ & $\geq 13.92$ & $13.29_{-0.09}^{+0.05}$ \\
\hline \multirow[t]{2}{*}{ NGC 3516} & $-197,-125$ & $\mathrm{HVC}$ & $<19.44$ & $12.98_{-0.15}^{+0.17}$ & $\geq 13.26$ & $12.86_{-0.19}^{+0.13}$ \\
\hline & $-90,-30$ & IVC & $19.83 \pm 0.06$ & $\geq 14.59$ & $\geq 13.74$ & $13.28_{-0.07}^{+0.11}$ \\
\hline \multirow[t]{4}{*}{ PG $1116+215$} & $-90,-25$ & IVC & $19.83_{-0.07}^{+0.06}$ & $\geq 14.44$ & $\geq 13.80$ & $13.24_{-0.08}^{+0.06}$ \\
\hline & 25,90 & IVC & $19.13_{-0.66}^{+0.25}$ & $13.42_{-0.09}^{+0.04}$ & $\geq 13.61$ & $12.59_{-0.15}^{+0.09}$ \\
\hline & 90,130 & HVC & $<19.49$ & $<12.22$ & $12.70_{-0.04}^{+0.11}$ & $12.39_{-0.09}^{+0.15}$ \\
\hline & 130,220 & HVC & $<19.49$ & $13.58_{-0.02}^{+0.02}$ & $\geq 13.44$ & $13.10_{-0.03}^{+0.03}$ \\
\hline \multirow[t]{3}{*}{ NGC 3783} & 105,158 & HVC & $\geq 20.00$ & $13.14_{-0.06}^{+0.11}$ & $12.95_{-0.12}^{+0.05}$ & $12.20_{-0.06}^{+0.12}$ \\
\hline & 158,208 & HVC & $19.18 \pm 0.70$ & $13.05_{-0.05}^{+0.00}$ & $12.58_{-0.03}^{+0.04}$ & $<11.59$ \\
\hline & 208,293 & HVC & $20.07 \pm 0.90$ & $14.27_{-0.02}^{+0.02}$ & $12.86_{-0.02}^{+0.04}$ & $12.64_{-0.01}^{+0.09}$ \\
\hline NGC 4051 & $-90,-50$ & IVC & $19.34_{-0.14}^{+0.11}$ & $\geq 14.45$ & $\geq 13.76$ & $13.20_{-0.11}^{+0.01}$ \\
\hline NGC 4151 & $-90,-50$ & IVC & $19.42_{-0.16}^{+0.14}$ & $\geq 14.67$ & $\overline{\geq} 13.57$ & $12.90_{-0.19}^{+0.16}$ \\
\hline \multirow[t]{3}{*}{ PG $1211+14$} & 35,90 & IVC & $<19.49$ & $13.41_{-0.08}^{+0.13}$ & $12.87_{-0.08}^{+0.18}$ & $12.07_{-0.16}^{+0.19}$ \\
\hline & 135,185 & HVC & $<19.49$ & $12.81_{-0.18}^{+0.12}$ & $12.36_{-0.05}^{+0.12}$ & $<11.76$ \\
\hline & 240,275 & HVC & $<19.49$ & $<12.13$ & $12.09_{-0.10}^{+0.05}$ & $<11.76$ \\
\hline \multirow[t]{2}{*}{ PG $1216+09$} & 148,219 & $\mathrm{HVC}$ & $<19.48$ & $<12.73$ & $12.87_{-0.07}^{+0.09}$ & $12.99_{-0.11}^{+0.14}$ \\
\hline & 219,287 & HVC & $<19.48$ & $<12.73$ & $\geq 13.20$ & $<12.35$ \\
\hline \multirow[t]{2}{*}{ Mrk 205} & $-225,-175$ & HVC & $19.44_{-0.19}^{+0.13}$ & $12.87_{-0.14}^{+0.11}$ & $12.43_{-0.17}^{+0.16}$ & $<12.11$ \\
\hline & $-175,-110$ & HVC & $18.11_{-0.11}^{+0.09}$ & $13.35_{-0.05}^{+0.06}$ & $13.22_{-0.07}^{+0.10}$ & $12.47 \pm 0.15$ \\
\hline $3 \mathrm{C} 273$ & 10,70 & IVC & $19.31_{-0.34}^{+0.11}$ & $14.15_{-0.18}^{-0.05}$ & $\begin{array}{l}\geq 13.77 \\
-0.07\end{array}$ & $13.13_{-0.14}^{+0.07}$ \\
\hline \multirow[t]{2}{*}{ Q $1230+011$} & 80,141 & $\mathrm{HVC}$ & $<19.67$ & $13.00_{-0.10}^{+0.18}$ & $12.61 \pm 0.07$ & $<11.93$ \\
\hline & 274,307 & HVC & $<19.67$ & $13.41_{-0.11}^{+0.10}$ & $12.54 \pm 0.07$ & $<12.33$ \\
\hline \multirow[t]{3}{*}{ NGC 4593} & 20,75 & IVC & $19.14_{-0.68}^{+0.25}$ & $13.83_{-0.28}^{+0.11}$ & $\geq 13.54$ & $13.01_{-0.21}^{+0.13}$ \\
\hline & 82,112 & HVC & $<19.52$ & $12.83_{-0.10}^{+0.28}$ & $12.43_{-0.08}^{+0.12}$ & $<12.23$ \\
\hline & 252,285 & HVC & $<19.52$ & $13.06 \pm 0.10$ & $12.29_{-0.01}^{+0.08}$ & $<12.50$ \\
\hline \multirow[t]{2}{*}{ PG $1259+59$} & $-155,-95$ & HVC & $19.37_{-0.42}^{+0.21}$ & $14.05_{-0.13}^{+0.14}$ & $\begin{array}{l}\geq 13.60 \\
-0.01\end{array}$ & $12.67_{-0.07}^{+0.06}$ \\
\hline & $-80,-30$ & IVC & $19.51_{-0.27}^{+0.16}$ & $\geq 14.77$ & $\geq 13.83$ & $12.92 \pm 0.06$ \\
\hline PKS 1302-102 & 200,340 & HVC & $<19.56$ & $<12.48$ & $12.23_{-0.15}^{+0.17}$ & $12.80_{-0.16}^{+0.19}$ \\
\hline Mrk 279 & $-226,-110$ & HVC & $19.28_{-0.50}^{+0.23}$ & $13.94_{-0.10}^{+0.08}$ & $\geq 14.05$ & $12.57_{-0.22}^{+0.16}$ \\
\hline & $-100,-55$ & IVC & $19.66_{-0.15}^{+0.11}$ & $\geq 14.54$ & $\geq 13.65$ & $13.29_{-0.14}^{+0.10}$ \\
\hline NGC 5548 & $-90,-25$ & IVC & $19.19_{-0.69}^{+0.15}$ & $\geq 14.84$ & $\geq 13.74$ & $13.68 \pm 0.05$ \\
\hline Mrk 1383 & $-90,-55$ & IVC & $\begin{array}{l}<19.43 \\
-0.69\end{array}$ & $13.53_{-0.20}^{+0.15}$ & $\geq 13.50$ & $13.00_{-0.11}^{+0.20}$ \\
\hline PG $1444+407$ & $-184,-126$ & HVC & $<19.50$ & $<12.60$ & $12.62_{-0.07}^{+0.08}$ & $<12.21$ \\
\hline & $-107,-77$ & IVC & $<19.50$ & $13.43_{-0.08}^{+0.10}$ & $\geq 13.55$ & $12.66_{-0.22}^{+0.18}$ \\
\hline & $-77,-37$ & IVC & $19.43_{-0.22}^{+0.14}$ & $\geq 14.52$ & $\geq 13.68$ & $13.29_{-0.06}^{+0.22}$ \\
\hline Mrk 876 & $-230,-155$ & HVC & $18.91_{-1.1}^{+0.28}$ & $13.67_{-0.07}^{+0.09}$ & $\geq 13.61$ & $12.56_{-0.08}^{+0.07}$ \\
\hline
\end{tabular}


TABLE $2-$ Continued

\begin{tabular}{|c|c|c|c|c|c|c|}
\hline Sightline & $\begin{array}{l}\text { Velocity Range } \\
\left(\mathrm{km} \mathrm{s}^{-1}\right)\end{array}$ & $\begin{array}{l}\text { Absorber } \\
\text { Type }\end{array}$ & $\begin{array}{c}\log N_{\mathrm{HI}} \\
\left(\mathrm{N} \text { in } \mathrm{cm}^{-2}\right)\end{array}$ & $\begin{array}{c}\log N_{\mathrm{SiII}} \\
\left(\mathrm{N} \text { in } \mathrm{cm}^{-2}\right)\end{array}$ & $\begin{array}{c}\log N_{\text {SiIII }} \\
\left(\mathrm{N} \text { in } \mathrm{cm}^{-2}\right)\end{array}$ & $\begin{array}{c}\log N_{\mathrm{SiIV}} \\
\left(\mathrm{N} \text { in } \mathrm{cm}^{-2}\right)\end{array}$ \\
\hline & $-155,-90$ & HVC & $18.97_{-0.71}^{+0.26}$ & $14.27_{-0.04}^{+0.07}$ & $\geq 13.92$ & $13.20_{-0.03}^{+0.06}$ \\
\hline HS $1700+6416$ & $-187,-85$ & HVC & $19.23_{-0.45}^{+0.22}$ & $\geq 14.36$ & $\geq 14.03$ & $13.13_{-0.12}^{+0.03}$ \\
\hline \multirow[t]{3}{*}{$3 \mathrm{C} 351$} & $-246,-183$ & HVC & $<19.61$ & $13 . \overline{1} 1 \pm 0.07$ & $\geq 13.50$ & $12.69_{-0.08}^{+0.09}$ \\
\hline & $-183,-138$ & HVC & $<19.61$ & $13.38_{-0.01}^{+0.11}$ & $12.83_{-0.12}^{+0.22}$ & $<12.07$ \\
\hline & $-138,-83$ & HVC & $<19.61$ & $13.87_{-0.08}^{+0.01}$ & $\geq 13.72$ & $12.94_{-0.06}^{+0.08}$ \\
\hline H $1821+643$ & $-188,-98$ & HVC & $<19.66$ & $14.15_{-0.06}^{+0.08}$ & $\geq 14.10$ & $13.42_{-0.05}^{+0.03}$ \\
\hline \multirow[t]{4}{*}{ Mrk 509} & $-343,-261$ & HVC & $<19.66$ & $<12.58$ & $13 . \overline{3} 0 \pm 0.12$ & $13.27 \pm 0.03$ \\
\hline & $-261,-218$ & HVC & $<19.66$ & $<12.58$ & $12.47_{-0.07}^{+0.08}$ & $12.34_{-0.01}^{+0.10}$ \\
\hline & 35,90 & IVC & $19.55_{-0.25}^{+0.16}$ & $14.74_{-0.08}^{+0.07}$ & $\geq 13.76$ & $13.46_{-0.08}^{+0.01}$ \\
\hline & 100,147 & HVC & $<19.66$ & $12.74_{-0.15}^{+0.12}$ & $12.70_{-0.12}^{+0.09}$ & $<12.47$ \\
\hline IR $2121-1757$ & 30,80 & IVC & $<19.76$ & $13.86_{-0.11}^{+0.19}$ & $\geq 13.84$ & $\geq 13.68$ \\
\hline \multirow[t]{4}{*}{ PHL 1811} & $-390,-333$ & HVC & $<19.48$ & $<12.27$ & $12.51 \pm 0.04$ & $12.58_{-0.05}^{+0.07}$ \\
\hline & $-314,-242$ & $\mathrm{HVC}$ & $<19.48$ & $<12.27$ & $13.07_{-0.03}^{+0.07}$ & $12.77_{-0.07}^{+0.06}$ \\
\hline & $-242,-190$ & $\mathrm{HVC}$ & $<19.48$ & $13.39 \pm 0.03$ & $\geq 13.38$ & $12.95 \pm 0.06$ \\
\hline & $-190,-133$ & HVC & $<19.48$ & $12.69_{-0.09}^{+0.08}$ & $\geq 13.43$ & $13.51_{-0.02}^{+0.03}$ \\
\hline \multirow[t]{2}{*}{ PKS 2155-304 } & $-314,-220$ & HVC & $<19.47$ & $<12.17$ & $12.33_{-0.04}^{+0.05}$ & $11.98_{-0.15}^{+0.15}$ \\
\hline & $-200,-96$ & $\mathrm{HVC}$ & $<19.47$ & $12.51_{-0.13}^{+0.16}$ & $13.17 \pm 0.02$ & $12.69_{-0.03}^{+0.04}$ \\
\hline \multirow[t]{4}{*}{ UGC 12163} & $-480,-390$ & HVC & $<19.69$ & $13.64_{-0.14}^{+0.13}$ & $\geq 13.91$ & $<12.54$ \\
\hline & $-390,-330$ & HVC & $<19.69$ & $<12.83$ & $12.95 \pm 0.30$ & $<12.84$ \\
\hline & $-185,-135$ & HVC & $<19.69$ & $<13.09$ & $\geq 13.16$ & $<12.84$ \\
\hline & $-90,-35$ & IVC & $19.60_{-0.23}^{+0.15}$ & $\geq 14.47$ & $\geq 13.75$ & $13.36_{-0.27}^{+0.41}$ \\
\hline \multirow[t]{4}{*}{ NGC 7469} & $-419,-279$ & HVC & $<19.47$ & $13 . \overline{5} 1 \pm 0.08$ & $\geq 13.91$ & $13.30_{-0.07}^{+0.07}$ \\
\hline & $-279,-246$ & HVC & $<19.47$ & $<12.75$ & $\geq 13.03$ & $12.75_{-0.12}^{+0.15}$ \\
\hline & $-199,-123$ & HVC & $<19.47$ & $<12.78$ & $12.54_{-0.12}^{+0.15}$ & $12.87_{-0.12}^{+0.12}$ \\
\hline & $-70,-20$ & IVC & $19.21_{-0.41}^{+0.21}$ & $\geq 14.65$ & $\geq 13.38$ & $13.16_{-0.08}^{+0.17}$ \\
\hline
\end{tabular}

a Several velocity ranges are selected for many sight lines, each treated as a separate HVC or IVC.

TABLE 3

HVC and IVC Model Parameters ${ }^{\mathrm{a}}$

\begin{tabular}{|c|c|c|c|c|c|}
\hline Sight line & Velocity Range & Absorber & $\log N_{\mathrm{HI}}$ & $\log U$ & $\log \left(Z / Z_{\odot}\right)$ \\
\hline \multirow[t]{5}{*}{$\operatorname{Mrk} 335^{\mathrm{a}}$} & $-426,-380$ & HVC & 19.4 & $\ldots$ & $\ldots$ \\
\hline & $-360,-275$ & HVC & 19.4 & $\ldots$ & $\ldots$ \\
\hline & $-257,-236$ & HVC & 19.4 & $\ldots$ & $\ldots$ \\
\hline & $-132,-80$ & HVC & 19.4 & $\ldots$ & $\ldots$ \\
\hline & $-80,-25$ & IVC & 19.5 & $-3.57,-3.41$ & $-0.77,-0.37$ \\
\hline \multirow[t]{2}{*}{ Ton S210 } & $-266,-221$ & HVC & $19.6,19.7$ & $\ldots$ & \\
\hline & $-221,-148$ & HVC & $19.6,19.7$ & $\cdots$ & $\cdots$ \\
\hline \multirow[t]{2}{*}{ HE0226-4410 } & 50,90 & IVC & 18.0 & $\ldots$ & $\ldots$ \\
\hline & 119,215 & HVC & 18.0 & $-2.07,-1.93$ & $-2.50,-1.83$ \\
\hline \multirow[t]{2}{*}{ PKS 0312-770 } & 70,280 & HVC & 18.0 & $-4.08,-3.62$ & $-2.50,-0.22$ \\
\hline & 280,372 & HVC & 18.0 & $-2.93,-2.82$ & $-2.50,-1.48$ \\
\hline PKS 0405-123 & 100,161 & HVC & 19.5 & & \\
\hline \multirow[t]{2}{*}{ NGC $1705^{\mathrm{a}}$} & 100,148 & HVC & 19.5 & $\ldots$ & $\ldots$ \\
\hline & 220,367 & HVC & 19.5 & $-3.50,-3.32$ & $-1.00,-0.61$ \\
\hline \multirow[t]{3}{*}{ HSO624+6907 } & $-150,-90$ & HVC & 19.2 & & \\
\hline & $-90,-60$ & IVC & 19.6 & $-3.42,-3.06$ & $-2.5,-0.87$ \\
\hline & 100,135 & $\mathrm{HVC}$ & 19.6 & $\ldots$ & $\ldots$ \\
\hline \multirow[t]{4}{*}{$\mathrm{PG} 0953+415^{\mathrm{a}}$} & $-171,-130$ & HVC & 19.3 & $\ldots$ & $\ldots$ \\
\hline & $-130,-100$ & HVC & 19.3 & $\ldots$ & $\ldots$ \\
\hline & 85,165 & HVC & 19.3 & $\ldots$ & $\cdots$ \\
\hline & 165,195 & HVC & 19.3 & $\ldots$ & $\ldots$ \\
\hline Ton 28 & 97,183 & HVC & 19.5 & $\ldots$ & $\ldots$ \\
\hline $3 \mathrm{C} 249$ & $-90,-30$ & IVC & 19.7 & $-3.42,-3.19$ & $-0.97,-0.46$ \\
\hline \multirow[t]{2}{*}{ NGC $3516^{\mathrm{a}}$} & $-187,-115$ & HVC & $19.4,19.5$ & $-2.73,-2.39$ & $-2.50,-2.18$ \\
\hline & $-90,-30$ & IVC & 19.8 & $-3.61,-2.19$ & $-2.50,-0.00$ \\
\hline \multirow[t]{4}{*}{$\mathrm{PG} 1116+215^{\mathrm{a}}$} & $-90,-25$ & IVC & 19.8 & $-3.36,-3.10$ & $-1.23,-0.62$ \\
\hline & 25,90 & IVC & 19.8 & $\ldots$ & $\ldots$ \\
\hline & 90,125 & HVC & 19.1 & $\ldots$ & $\ldots$ \\
\hline & 130,220 & HVC & 19.5 & $-2.83,-2.76$ & $-2.50,-1.82$ \\
\hline \multirow[t]{3}{*}{ NGC 3783} & 100,153 & HVC & 18.3 & $\ldots$ & $\ldots$ \\
\hline & 153,203 & HVC & 18.0 & $\ldots$ & $\ldots$ \\
\hline & 203,288 & $\mathrm{HVC}$ & 19.2 & $\ldots$ & $\ldots$ \\
\hline NGC 4051 & $-90,-30$ & IVC & $19.6,19.7$ & $-3.49,-2.30$ & $-2.27,-0.00$ \\
\hline NGC 4151 & $-90,-50$ & IVC & 19.4 & $-3.79,-2.50$ & $-2.50,-0.00$ \\
\hline
\end{tabular}


TABLE 3 - Continued

\begin{tabular}{|c|c|c|c|c|c|}
\hline Sight line & Velocity Range & Absorber & $\log N_{\mathrm{HI}}$ & $\log U$ & $\log \left(Z / Z_{\odot}\right)$ \\
\hline \multirow[t]{3}{*}{$\mathrm{PG} 1211+143^{\mathrm{a}}$} & 47,90 & IVC & 19.5 & $\cdots$ & $\cdots$ \\
\hline & 155,208 & HVC & 19.5 & $\ldots$ & $\ldots$ \\
\hline & 260,293 & HVC & 19.5 & $\ldots$ & $\ldots$ \\
\hline \multirow[t]{2}{*}{ PG 1216+069 } & 165,234 & HVC & 19.5 & $\ldots$ & $\ldots$ \\
\hline & 234,302 & HVC & 19.5 & $-3.01,-2.67$ & $-2.50,-2.16$ \\
\hline \multirow[t]{2}{*}{$\operatorname{Mrk} 205^{\mathrm{a}}$} & $-225,-175$ & HVC & $19.4,19.5$ & $\ldots$ & \\
\hline & $-175,-110$ & $\mathrm{HVC}$ & 18.1 & $-3.15,-3.05$ & $-2.50,-1.91$ \\
\hline $3 \mathrm{C} 273$ & 10,70 & IVC & 19.3 & $-3.27,-3.08$ & $-1.59,-0.82$ \\
\hline \multirow{2}{*}{$\mathrm{Q} 1230+0115^{\mathrm{a}}$} & 95,152 & HVC & 19.6 & $\ldots$ & $\ldots$ \\
\hline & 285,318 & HVC & 19.6 & $\ldots$ & $\ldots$ \\
\hline \multirow[t]{3}{*}{ NGC $4593^{\mathrm{a}}$} & 20,75 & IVC & $19.1,19.2$ & $-3.24,-2.82$ & $-2.50,-1.07$ \\
\hline & 88,118 & HVC & 19.5 & $\ldots$ & $\ldots$ \\
\hline & 258,291 & HVC & 19.5 & $\ldots$ & $\ldots$ \\
\hline \multirow[t]{2}{*}{ PG $1259+593$} & $-180,-105$ & HVC & 19.4 & $-3.47,-3.24$ & $-1.83,-0.94$ \\
\hline & $-80,-30$ & IVC & 19.5 & $-3.41,-2.82$ & $-1.15,-0.00$ \\
\hline PKS 1302-102 & 200,340 & HVC & $<19.56$ & $\ldots$ & $\ldots$ \\
\hline \multirow[t]{2}{*}{ Mrk 279} & $-226,-115$ & HVC & $19.2,19.3$ & $\ldots$ & $\ldots$ \\
\hline & $-105,-55$ & IVC & 19.7 & $-3.50,-3.13$ & $-1.08,-0.22$ \\
\hline NGC 5548 & $-80,-25$ & IVC & $19.1,19.2$ & $-3.80,-2.45$ & $-2.50,-0.00$ \\
\hline Mrk 1383 & $-90,-55$ & IVC & 19.4 & $-3.03,-2.67$ & $-2.50,-1.68$ \\
\hline \multirow[t]{3}{*}{$\mathrm{PG} 1444+407^{\mathrm{a}}$} & $-164,-109$ & HVC & 19.5 & $\ldots$ & $\ldots$ \\
\hline & $-109,-60$ & IVC & 19.5 & $\ldots$ & $\ldots$ \\
\hline & $-60,-20$ & IVC & 19.4 & $-3.71,-2.39$ & $-2.50,-0.00$ \\
\hline \multirow[t]{2}{*}{ Mrk $876^{\mathrm{a}}$} & $-210,-155$ & HVC & $18.9,19.0$ & $-3.42,-3.31$ & $-2.32,-1.10$ \\
\hline & $-155,-90$ & $\mathrm{HVC}$ & 19.0 & $-3.34,-3.26$ & $-1.52,-0.5$ \\
\hline \multirow{4}{*}{$\begin{array}{l}\text { HS1700+6416 } \\
3 \mathrm{C} 351^{\mathrm{a}}\end{array}$} & $-197,-85$ & HVC & $19.2,19.3$ & $-3.42,-3.20$ & $-1.28,-0.52$ \\
\hline & $-221,-150$ & HVC & 19.6 & & \\
\hline & $-150,-107$ & HVC & 19.6 & $-3.11,-3.08$ & $-2.50,-1.98$ \\
\hline & $-107,-45$ & IVC & 19.6 & $-3.19,-2.99$ & $-2.50,-1.24$ \\
\hline \multirow{5}{*}{$\begin{array}{l}\text { H } 1821+643 \\
\text { Mrk } 509^{\mathrm{a}}\end{array}$} & $-165,-75$ & HVC & $19.6,19.7$ & $-3.00,-2.78$ & $-2.50,-1.0$ \\
\hline & $-338,-256$ & HVC & $19.6,19.7$ & $\ldots$ & $\ldots$ \\
\hline & $-256,-213$ & HVC & $19.6,19.7$ & $\ldots$ & $\ldots$ \\
\hline & 35,90 & IVC & $19.5,19.6$ & $-3.31,-3.19$ & $-0.92,-0.42$ \\
\hline & 100,147 & HVC & $19.6,19.7$ & $\ldots$ & \\
\hline \multirow{5}{*}{$\begin{array}{l}\text { IR } 2121-1757 \\
\text { PHL } 1811^{\mathrm{a}}\end{array}$} & 30,80 & IVC & $19.7,19.8$ & $-3.42,-3.20$ & $-1.28,-0.52$ \\
\hline & $-385,-328$ & $\mathrm{HVC}$ & 19.5 & & \\
\hline & $-309,-237$ & HVC & $19.5,19.5$ & $\ldots$ & $\cdots$ \\
\hline & $-237,-180$ & HVC & 19.5 & $-2.79,-2.54$ & $-2.50,-2.02$ \\
\hline & $-180,-128$ & HVC & 19.5 & $\ldots$ & $\ldots$ \\
\hline \multirow[t]{2}{*}{ PKS 2155-304 } & $-309,-215$ & HVC & 19.5 & $\ldots$ & $\ldots$ \\
\hline & $-195,-91$ & HVC & 19.5 & $\ldots$ & $\ldots$ \\
\hline \multirow[t]{4}{*}{$\mathrm{UGC} 1216^{\mathrm{a}}$} & $-477,-381$ & HVC & 19.7 & $-2.78,-2.73$ & $-2.50,-2.45$ \\
\hline & $-372,-324$ & HVC & 19.7 & $\ldots$ & $\ldots$ \\
\hline & $-172,-135$ & $\mathrm{HVC}$ & 19.7 & $\ldots$ & $\ldots$ \\
\hline & $-90,-35$ & IVC & 19.6 & $-3.50,-2.99$ & $-1.22,-0.15$ \\
\hline \multirow[t]{4}{*}{ NGC $7469^{\mathrm{a}}$} & $-410,-270$ & HVC & 19.5 & $-2.68,-2.56$ & $-2.50,-1.8$ \\
\hline & $-270,-237$ & HVC & 19.5 & $\ldots$ & $\ldots$ \\
\hline & $-190,-114$ & HVC & 19.5 & $\ldots$ & $\ldots$ \\
\hline & $-60,-15$ & IVC & 19.4 & $-2.98,-2.68$ & $-2.17,-1.47$ \\
\hline
\end{tabular}

${ }^{\mathrm{a}}$ Columns list ranges of model parameters, $\log N_{\mathrm{HI}}, \log U$, and $\log \left(Z / Z_{\odot}\right)$. There are multiple velocity ranges for these sight lines, each treated as a separate HVC or IVC. 